\title{
BIFURCATION ANALYSIS FOR A CLASS OF PROBLEMS WITH A FREE BOUNDARY
}

\author{
JAN SIJBRAND \\ University of Utrecht. Mathematical Institute, Budapestlaan 6, Utrecht 2506, Holland
}

(Received 20 November 1978)

Key words: Nonlinear elliptic PDE, free boundary, domain perturbation, Lyapunov-Schmidt method, bifurcation, confined plasma.

\section{INTRODUCTION}

IN THIS PAPER we study problems in a bounded region in $\mathbf{R}^{n}$ which is divided into two subregions, separated by a free boundary. We search for a function $u$, defined on the entire domain, which satisfies a different elliptic differential equation on each subdomain and an appropriate smoothness condition on the free boundary.

A major application of our analysis is the construction of equilibrium solutions for the equations describing a plasma in a cavity. For certain values of physical parameters this plasma will not fill the entire cavity but will be concentrated in a small subdomain. The aim of physical experiments of this kind is to obtain very high concentrations of plasma inside a toroidal tube (the 'Tokamak' machine) and very high temperatures such that a nuclear fusion reaction is ignited (see Mercier [1]).

The free boundary problem has recently received much attention (Temam [2, 3], Schaeffer [4], Puel [5], Kinderlehrer and Spruck [6, 7]). For the problem in $\mathbf{R}^{2}$, Temam has proved the existence of a unique solution for a limited range of physical parameters. For a very special configuration in $\mathbf{R}^{2}$, Schaeffer proved the existence of multiple solutions for sets of parameters outside Temam's range; these solutions may be constructed numerically.

In this paper we shall analyse the set of equilibrium solutions using the techniques of the theory of bifurcation. The main features of our approach are: (1) solutions are constructed analytically, (2) many bifurcation points are found, which lead to the same number of equilibrium solutions, (3) the bifurcation points do not coincide with the eigenvalues of the elliptic operator on the big domain.

Our analysis is, in its main lines, conceptually not very difficult. However, working out all steps in full rigour, and all details in full technical complexity, tends to obscure the main line of reasoning. This is why the presentation of the paper is organized as follows.

In Section 1 we formulate the free boundary problem and give a descriptive outline of the method of solution.

In Section 2, in order to convince the reader that the method works, we describe how a special class of problems can explicitly be tackled and, as an example, we analyse fully a cylindrical domain in $\mathbf{R}^{3}$.

In Section 3, we finally give a rigorous treatment of the original general problem.

Various lengthy computational details, of which the reading is not strictly necessary for the understanding of this paper, are presented separately in Ref. [8]. 


\section{SKETCH OF THE PROBLEM AND OF THE METHOD OF SOLUTION}

The treatment in this section is descriptive. Our aim is to sketch the method of analysis omitting as far as possible the technical details. The reader who is concerned about the validity of certain arguments is referred to Section 3.

Let us consider a domain $\Omega \subset \mathbf{R}^{n}$. We want to find a function $u$ and a subdomain $\omega$ defined by $\omega=\{x \in \Omega: u(x)>0\}$ such that

in $\omega$ :

on $\partial \omega$ :

in $\Omega \backslash \omega$ :

on $\partial \Omega$ :

$\operatorname{across} \partial \omega$ :

$$
\begin{aligned}
\mathscr{L} u+\lambda u & =0, \\
u & =0, \\
\mathscr{L} u & =0, \\
u & =\text { constant }-1,
\end{aligned}
$$

$\mathscr{L}$ is a uniformly clliptic operator of the form

$$
\mathscr{L}=\sum_{i, j} \frac{\partial}{\partial x_{j}} \alpha_{i j}(x) \frac{\partial}{\partial x_{j}} ;
$$

$\lambda$ is a positive real constant; $\partial / \partial n$ is the normal derivative on $\partial \omega ; \alpha_{i j}=\alpha_{j i}$.

Let $\mu_{i}$ be the $i^{\text {th }}$ eigenvalue of $\mathscr{L}$ on $\Omega$ with zero boundary conditions, the $\mu_{i}$ being numbered according to magnitude, multiplicities taken into account. If $\lambda \leqslant \mu_{1}, u=-1$ is the only solution of (1.1-1.5). A less trivial result was found for $\mathscr{L}=\Delta$ by Temam [3] $(n=2)$ and by Puel [5] $(n$ arbitrary): if $\mu_{1}<\lambda \leqslant \mu_{2}$, then there is exactly one non-constant solution (with a non-empty $\omega$ ).

We shall now investigate what happens if $\lambda$ increases beyond $\mu_{2}$. We know (see Berestycki and Brézis [9]) that the solution branch which exists for $\lambda \leqslant \mu_{2}$ can be continued, so we have a branch of solutions

$$
\mathscr{B} \mathscr{B}=\left\{\left(\omega_{0}(\lambda), u_{0}(\lambda), \lambda\right)\right\}
$$

Now suppose that there is a number $\lambda_{0}$, with $\left(\omega_{0}\left(\lambda_{0}\right), u_{0}\left(\lambda_{0}\right), \lambda_{0}\right) \in \mathscr{B} \mathscr{B}$ while there are solutions of $(1.1-1.5),(\tilde{\omega}, \tilde{u}, \lambda)$ that are in a sense close to $\left(\omega_{0}\left(\lambda_{0}\right), u_{0}\left(\lambda_{0}\right), \lambda_{0}\right)$ but not on $\mathscr{B} \mathscr{B}$. Then $\lambda_{0}$ represents a 'bifurcation point' of $\mathscr{B} \mathscr{B}$. Because $\tilde{\omega}$ is 'close' to $\omega_{0}\left(\lambda_{0}\right)$, we may represent the boundary of $\tilde{\omega}$ by a function $\tilde{\gamma}: \partial \omega_{0} \rightarrow \mathbf{R}^{n}$, as follows:

$$
\tilde{\gamma}=1+\varepsilon \rho \hat{n},
$$

where 1 is the identity function on the boundary of $\omega_{0}\left(\lambda_{0}\right), \hat{n}$ is the unit vector normal to this boundary, $\varepsilon$ is a small parameter fixing the 'size' of the perturbation and $\rho$ is a real function, of order unity, which represents the deformation of the boundary from $\omega_{0}\left(\lambda_{0}\right)$ to $\bar{\omega}$. Of course, at this stage the shape of $\rho$ is unknown, and we have not yet given an unambiguous method for determining the 'size' and 'shape' of a given small perturbation; we shall return to this later in the section. At this moment, $\rho$ is a smooth mapping from $\partial \omega_{0}$ to $\mathbf{R}$. In Section 3, more attention will be given to the class of admissible boundary perturbations $\rho$.

Now let $T(\varepsilon, \rho)$ be a coordinate transformation which maps $\omega_{0}\left(\lambda_{0}\right)$ to $\tilde{\omega}$. We suppose

$$
T(\varepsilon, \rho)=\sum_{n \geqslant 0} \varepsilon^{n} T_{n}(\rho)
$$


Applying this transformation to (1.1-1.2) together with the definition of $\omega$, we obtain

$$
\begin{aligned}
L U+\lambda U=0 & \text { in } \omega_{0}, \\
U=0 & \text { on } \partial \omega_{0} \\
U>0 & \text { in } \omega_{0},
\end{aligned}
$$

where $L$ is the operator $\mathscr{L}$ after $T$ has been applied; we expect

$$
L=\sum_{n \geqslant 0} \varepsilon^{n} L_{n}(\rho)
$$

where the $L_{n}$ are again second order differential operators. The solution $u$ of (1.9) is the first eigenfunction of $L$ on $\omega_{0}$ and we expect it to have a power series representation in $\delta:$

$$
U=\sum_{n \geqslant 0} \varepsilon^{n} U_{n}(\rho)
$$

and also

$$
\lambda=\sum_{n \geqslant 0} \varepsilon^{n} \lambda_{n}(\rho)
$$

where the $U_{n}(\rho)$ are functions on $\omega_{0}$ and the $\lambda_{n}(\rho)$ are real constants;

$$
U_{0}=u_{0}
$$

where $u_{0}$ is defined in (1.6) and $\lambda_{0}$ is the supposed bifurcation point. By equating powers of $\varepsilon$ we may obtain the coefficients $T_{n}, L_{n}, U_{n}$ and $\lambda_{n}$; of course they still depend on the arbitrary perturbation shape $\rho$.

If we define $u_{i n}(\varepsilon, \rho, x)=U\left(T^{-1}(\varepsilon, \rho) x\right)$ for $x$ in $\tilde{\omega}$ with $U$ the solution of $(1.9)$, then $u_{i n}$ satisfies (1.1-1.2). We now turn to all $\Omega$. We define a new problem: let $u_{\text {pre }}$ satisfy

$$
\begin{aligned}
\mathscr{L} u_{\text {pre }}+\lambda u_{\text {in }}=0 & & \text { in } \tilde{\omega}, \\
\mathscr{L} u_{\text {pre }}=0 & & \text { in } \Omega \backslash \tilde{\omega}, \\
u_{\text {pre }}=0 & & \text { on } \partial \Omega .
\end{aligned}
$$

Of course, $u_{\text {pre }}$ depends (through $\left.\lambda, u_{i n}, \tilde{\omega}\right)$ on $\varepsilon$ and $\rho$. If now $\rho(\varepsilon)$ can be chosen such that for small $\varepsilon, u_{\text {pre }}$ is a constant $\alpha(\varepsilon)$ on $\partial \tilde{\omega}$, then $u_{\text {pre }}-\alpha(\varepsilon)=u_{\text {in }}$ inside $\tilde{\omega}$ and we find that $u_{\text {pre }}-\alpha(\varepsilon)$ satisfies $(1.1,1.2,1.3,1.5)$, hence

$$
\tilde{u}=\frac{u_{\mathrm{pre}}}{\alpha(\varepsilon)}-\alpha(\varepsilon)
$$

satisfies all conditions of (1.1-1.5), and with 1.12 and 1.7 we find a branch $(\tilde{\omega}(\varepsilon), \tilde{u}(\varepsilon), \lambda(\varepsilon))$ of solutions. Eventually, we can invert $\lambda(\varepsilon)$ to obtain $\varepsilon$ in terms of $\lambda-\lambda_{0}$; we then have the bifurcating branch in the form $\left(\tilde{\omega}\left(\lambda-\lambda_{0}\right), \tilde{u}\left(\lambda-\lambda_{0}\right), \lambda\right)$. Hence, if we define

$$
F(\varepsilon, \rho, x)=\lambda^{-1} u_{\text {pre }}(x)
$$

then the basic question is: can $\rho$ be chosen such that $F(\varepsilon, \rho, x)$ is a constant function $a(\varepsilon)$ for $x \in \partial \tilde{\omega}$ ? Equivalently, if we define

$$
f(\varepsilon, \rho, y)=F(\varepsilon, \rho, T(\varepsilon, \rho) y),
$$


for $y \in \partial \omega_{0}$, then finding a solution for (1.1-1.5) amounts to finding a $\rho(\varepsilon)$ such that

$$
f(\varepsilon, \rho, \cdot)=a(\varepsilon)
$$

identically on $\partial \omega_{0}$.

We now study $f: \rho \rightarrow f(\varepsilon, \rho,$.$) as an operator acting in the space of real functions on \partial \omega_{0}$. We develop $f$ in powers of $\varepsilon$ :

$$
f(\varepsilon, \rho, \cdot)=\sum_{n \geqslant 0} \varepsilon^{n} f_{n}(\rho)(\cdot)
$$

and expecting a power series in $\varepsilon$ for $\alpha(\varepsilon)$, we set $a(\varepsilon)=f_{0}+\varepsilon b(\varepsilon)$. So $f=$ constant means:

$$
f_{1} \rho=-\left(\varepsilon f_{2}(\rho)+\varepsilon^{2} f_{3}(\rho)+\ldots\right)+b(\varepsilon) .
$$

$f_{1}$ is a linear operator on the space of real functions on $\partial \omega_{0}$.

We now apply the usual bifurcation techniques (for an introduction, see e.g. Stakgold [10] and Sattinger [11]): if $f_{1}$ is invertible, then (1.19) has a unique solution $\rho(\varepsilon)$, but this solution represents the variation of the basic branch $\mathscr{B} \mathscr{B}$. Hence, $\lambda_{0}$ will be a bifurcation point only if $f_{1}$ is non-invertible, i.e. if a $\phi_{0}$ exists with $f_{1} \phi_{0}=0$. In this casc we decompose $\rho$ into a component along $\phi_{0}$ and a residual one, so we get

$$
\rho=\kappa(\varepsilon) \phi_{0}+\psi(\varepsilon)
$$

Hence,

$$
f_{1} \psi=-\varepsilon f_{2}\left(\kappa \phi_{0}+\psi\right)-\varepsilon^{2} f_{3}\left(\kappa \phi_{0}+\psi\right)+\ldots+b,
$$

which has a solution

$$
\psi=\tilde{f}_{1}^{-1}\left(-\varepsilon f_{2}\left(\kappa \phi_{0}+\psi\right)+\ldots+b\right)
$$

provided

$$
P\left(-\varepsilon f_{2}\left(\kappa \phi_{0}+\psi\right)+\ldots+b\right)=0,
$$

where $\tilde{f}_{1}^{-1}$ is the pseudoinverse on the $\psi$-space and $P$ is the projection onto $\phi_{0}^{*}$. From (1.22) we can obtain $\psi=\Psi(\varepsilon, b, \kappa)$; introducing this into (1.23) we obtain an equation for $\kappa, \varepsilon$ and $b$, which can be solved for $b$ in terms of $\varepsilon$ and $\kappa$. So $\Psi$ is known in terms of $\varepsilon$ and $\kappa$. If we now substitute $\Psi(\varepsilon, b(\varepsilon, \kappa), \kappa)$ into (1.20) and the expression obtained for $\rho(\varepsilon, \kappa)$ into $(1.12)$, we get a relation for $\lambda, \varepsilon$ and $\kappa$ which is typically of the form

$$
\lambda-\lambda_{0}=c_{1} \kappa \varepsilon+c_{2} \kappa^{2} \varepsilon^{2}+c_{3} \kappa^{3} \varepsilon^{3}+\ldots,
$$

where $c_{1}, c_{2}$ are real constants. If $c_{1} \neq 0$, a proper choice for $\varepsilon$ is $\varepsilon=\lambda-\lambda_{0}$. If $c_{1}=0, c_{2} \neq 0$ then we take $\varepsilon=\left|\lambda-\lambda_{0}\right|^{1 / 2}$, etc. (1.24) Now becomes an equation for $\kappa$ and $\lambda-\lambda_{0}$ alone, which is easily solved for $\kappa$ in terms of $\lambda-\lambda_{0}$. By (1.20), (1.11), (1.14) and (1.12) we then find a bifurcating solution of problem (1.1-1.5).

We summarize the analysis sketched in this section: if, for increasing $\lambda$ an eigenvalue of $f_{1}$ crosses 0 , we can expect bifurcation. If this happens for $\lambda=\lambda_{0}$, we calculate the coefficients $c_{1}, c_{2} \ldots$ until we find a non-vanishing one. If, for instance, we are in the situation $c_{1}=0$, $c_{2} \neq 0$, the bifurcating boundary perturbation function can be of the form

$$
\varepsilon \rho- \pm \sqrt{\frac{\lambda-\lambda_{0}}{c_{2}}} \phi_{0}+O\left(\lambda-\lambda_{0}\right),
$$


so we have a super- or subcritically bifurcating branch of free boundaries $\tilde{\gamma}$ (depending on sign $c_{2}$ ), with corresponding domains $\tilde{\omega}$ and functions

$$
\tilde{u}=\frac{u_{\mathrm{pre}}-\alpha}{\alpha} .
$$

\section{A SPECIAL CLASS OF PROBLEMS}

\subsection{Special geometries}

In this section we consider domains $\Omega$ and operators $\mathscr{L}$ which have certain symmetry properties. This will permit us to establish explicit results. Of course many interesting problems will not have the properties we now require, but we expect that phenomena which can be observed in our simple configurations will help clarify the behaviour in the more complex problems.

The geometrical configurations $\Omega$ we study now include the following cases:

(i) a circular domain in $\mathbf{R}^{2}$,

(ii) an infinite cylinder in $\mathbf{R}^{3}$, with $z$-periodic structure (i.e. we only study functions with prescribed period $2 \pi$ in the $z$-direction) and with a circular cross-section,

(iii) an infinite strip in $\mathbf{R}^{2}$, with the same periodic structure,

(iv) an annulus in $\mathbf{R}^{2}$

and generalizations to higher dimensions.

In all these (and other) cases, $\Omega$ can be parametrized by the variables $r$ and $\phi_{1}, \ldots, \phi_{n-1}$ which can vary in the ranges $r \in R=[0,1]$ and $\phi \in \Phi=[0,2 \pi)^{n-1}$ (e.g. for case (ii), if the cylinder is described by cylindrical coordinates $\left(r, \phi_{1}, z\right)$, the $z$ variable is renamed $\left.\phi_{2}\right)$. The boundary of $\Omega$ may be either $\{r=1\}$ (cases (i), (ii)) or $\{r=0\} \cup\{r=1\}$ (cases (iii), (iv)).

Later on in this section we shall derive explicit formulas by which we can compute directly the bifurcation values and the bifurcating free boundaries for the domains $\Omega$ with boundary $\{r=1\}$. We have picked out case (ii) as an example to obtain explicit results (Section 2.5.1).

If the boundary of $\Omega$ consists of $\{r=0\} \cup\{r=1\}$ we expect two free boundaries, which slightly complicates the calculations. To show that no essential difficulty arises, we have computed the bifurcation points for case (iii) (Section 2.5.2). The details of the calculations in this section are described in [8].

\subsection{Properties of $\Omega$ and $\mathscr{L}$}

On $\Omega$ an inner product is defined by

$$
(u, v)_{\Omega}=\int_{\Omega} u \cdot v \mathrm{~d} x=\int_{\substack{r \in R \\ \phi \in \Phi}} u(r, \phi) v(r, \phi) r^{f} Q(\phi) \mathrm{d} r \mathrm{~d} \phi,
$$

with $f$ a natural number, $0 \leqslant f \leqslant n-1$ and $Q$ a real function on $\Phi\left(r^{f} Q(\phi)\right.$ is the Jacobian belonging to the coordinates $(r, \phi))$. For 'separated' functions $u(r, \phi)=\xi_{1}(r) \theta_{1}(\phi), v(r, \phi)=$ $\xi_{2}(r) \theta_{2}(\phi)$ we can split the inner product:

$$
(u, v)_{\Omega}=\left(\xi_{1}, \xi_{2}\right)_{R}\left(\theta_{1}, \theta_{2}\right)_{\Phi}
$$


with

$$
\begin{aligned}
& \left(\xi_{1}, \xi_{2}\right)_{R}=\int_{r \in R} \xi_{1}(r) \xi_{2}(r) r^{f} \mathrm{~d} r \\
& \left(\theta_{1}, \theta_{2}\right)=\int_{\phi \in \Phi} \theta_{1}(\phi) \theta_{2}(\phi) Q(\phi) \mathrm{d} \phi .
\end{aligned}
$$

Let us further assume that in terms of the variables $(r, \phi)$, the operator $\mathscr{L}$ is of the form:

$$
\mathscr{L}(\xi(r) \Theta(\phi))=\Theta(\phi) \mathscr{L}_{r}(\xi(r))+\xi(r) \mathscr{L}_{\phi}(\Theta(\phi))
$$

where

$$
\mathscr{L}_{\phi}=h(r) D_{1}+D_{2}
$$

$h$ is a real function, $\mathscr{L}_{r}$ is a self adjoint operator in the variable $r$, independent of $\phi ; D_{1}$ is a self adjoint operator in the variables $\phi_{1} \ldots \phi_{k}$, independent of $r$ and $D_{2}$ is a self adjoint operator in the variables $\phi_{k+1} \ldots \phi_{n-1}$, independent of $r$.

$$
\begin{aligned}
& \mathscr{L}_{r}=\sum_{p=1}^{2} a_{p}(r) \frac{\partial^{p}}{\partial^{p} r}, \\
& D_{1}=\sum_{\substack{i=1 \\
1 \leqslant p_{i} \leqslant k}}^{2} b_{p_{1}} \cdots p_{p_{\ell}}\left(\phi_{1}, \ldots, \phi_{k}\right) \frac{\partial}{\partial \phi_{p_{1}}} \ldots \frac{\partial}{\partial \phi_{p r}} \\
& D_{2}=\sum_{\substack{\ell=1 \\
k+1 \leqslant p_{i} \leqslant n-1}}^{2} c_{p_{1} \ldots p \ell}\left(\phi_{k+1}, \ldots, \phi_{n-1}\right) \frac{\partial}{\partial \phi_{p_{1}}} \ldots \frac{\partial}{\partial \phi_{p}},
\end{aligned}
$$

$a_{p}, b_{p_{1} \ldots p f}$ and $c_{p_{1} \ldots p \text {, }}$ are real functions on $R, \Phi_{1}, \Phi_{2}$, respectively, where $\Phi_{1}=\operatorname{sp}\left(\phi_{1}, \ldots, \phi_{k}\right)$ and $\Phi_{2}=\operatorname{sp}\left(\phi_{k+1}, \ldots, \phi_{n-1}\right)$. The operator $\Delta$, expressed in spherical or cylindrical coordinates $(r, \phi)$ satisfies all the above requirements.

The eigenfunctions of $D_{1}$ and $D_{2}$ on $\Phi_{1,2}$ (with periodic boundary conditions) are $\Theta_{j_{1}}, \Theta_{j_{2}}$ $\left(j_{1,2}=0,1,2, \ldots\right)$, respectively:

$$
D_{i} \Theta_{j_{i}}+v_{j_{i}} \Theta_{j_{i}}=0, \quad(i=1,2)
$$

We now take these $\Theta$ 's together in the form $\Theta_{j}=\Theta_{j_{1}} . \Theta_{j_{2}}$ (to every $j=0,1,2 \ldots$ there belongs a unique pair $j_{1}, j_{2}$ ); for $\Theta_{j}$, we have

$$
D_{i} \Theta_{j}+v_{j_{i}} \Theta_{j}=0
$$

Because the $D_{1,2}$ contain no constant term there is a constant eigenfunction $\Theta_{0}$ with eigenvalue 0 . We assume the eigenfunctions $\Theta_{j}$ to be normalized:

$$
\delta_{j k}=\left(\Theta_{j}, \Theta_{k}\right)_{\Phi}
$$

Let $u=\xi_{i j}$ be the eigenfunctions and $\lambda=\mu_{i j}$ the eigenvalues of

$$
\begin{gathered}
\mathscr{L}_{r} u-h(r) v_{j_{1}} u-v_{j_{2}} u+\lambda u=0, \\
u=0 \text { on } \partial \Omega
\end{gathered}
$$


The eigenfunctions of $\mathscr{L}$ on $\Omega$ are then of the form

$$
E_{i j}(r, \phi)=\xi_{i j}(r) \Theta_{j}(\phi),
$$

with eigenvalues $\mu_{i j}$, while on a subdomain $\omega=R^{\prime} \times \Phi$ (with $R^{\prime} \subset R$ ) the eigenfunctions are of the form

$$
E_{i j}^{\prime}=\zeta_{i j}^{\left(R^{\prime}\right)}(r) \Theta_{j}(\phi)
$$

with eigenvalues $\mu_{i j}\left(R^{\prime}\right)$.

We assume the $\xi_{i j}$ to be normalized by

$$
\delta_{i k}=\left(\xi_{i j}, \xi_{k j}\right)_{R}
$$

(and a similar relation for the $\zeta_{i j}$ on $R^{\prime}$ ). Because $\mathscr{P}$ is self adjoint the $E_{i j}$ and $E_{i j}^{\prime}$ form a complete set on $\Omega, \omega$ respectively.

Let $\xi_{0}(r)$ be the positive eigenfunction of (2.4) with lowest eigenvalue $\left(j_{1}=j_{2}=0\right)$ :

$$
\mathscr{L}_{r} \xi_{0}(r)+\mu_{0} \xi_{0}(r)=0 \text {. }
$$

For every $\lambda>\mu_{0}$ there will be a unique domain $R_{\lambda} \subset R$ such that (1.1)-(1.5) is solved, with

$$
\begin{aligned}
\omega=R_{\lambda} \times \Phi & \\
\mathscr{L}_{r} u(r)+\lambda u(r) & =0 \text { in } \omega, \\
\mathscr{L}_{r} u(r) & =0 \text { in } \Omega \backslash \omega, \\
u & =-1 \text { on } \partial \Omega, \\
u & =0 \text { on } \partial \omega
\end{aligned}
$$

where, of course, $u(r)=\zeta_{0}^{\left(R_{\lambda}\right)}(r)$ inside $\omega$ and $\lambda=\mu_{0}\left(R_{\lambda}\right)$.

We further suppose

$$
\text { a } \begin{aligned}
\mathscr{L}_{r} & =\sum_{p=1}^{2} a_{p} r^{-g+p} \frac{\partial^{p}}{\partial^{p} r}, \text { for some } g \in \mathbf{N} \\
\text { and } \partial \Omega & =\{r=1\},
\end{aligned}
$$

then, due to (2.4) we have for $\lambda \geqslant \mu_{0}$ a branch of solutions of the form (1.8):

$$
\begin{aligned}
& \omega_{0}(\lambda)=\left\{(r, \phi): 0 \leqslant r<r_{0}(\lambda) ; \phi \in \Phi\right\} ; r_{0}(\lambda)=\left(\frac{\mu_{0}}{\lambda}\right)^{1 / g} \\
& u_{0}(\lambda)=\xi_{0}\left(\frac{r}{r_{0}}\right) \text { for } 0 \leqslant r<r_{0}
\end{aligned}
$$

and $\mu_{0}(\lambda)$ is extended to $r_{0} \leqslant r<1$ as the unique solution of

$$
\mathscr{L}_{r} u(r)=0 ; u\left(r_{0}\right)=0 ; u^{\prime}\left(r_{0}\right)=\frac{1}{r_{0}} \xi_{0}^{\prime}(1)
$$

\subsection{Computation of $f_{1}$; linear analysis}

Let us suppose that the boundary of the bifurcating domain $\tilde{\omega}$ can be represented by the function $\tilde{\gamma}: \Phi \rightarrow \mathbf{R} \times \Phi$ defined by

$$
\tilde{\gamma}(\phi)=\left(r_{0}(1+\varepsilon \rho(\phi)), \phi\right) .
$$


We develop the perturbation shape $\rho$ in terms of the orthonormal set of eigenfunctions defined on $\Phi$ :

$$
\rho(\phi)=\sum_{j} \gamma_{j} \Theta(\phi)
$$

The next step is to compute the operators $T_{n}(\rho)$ and $L_{n}(\rho)$ in terms of the function $\rho$ or its coefficients $\gamma_{j}$. For the transformation of coordinates $T(\varepsilon ; \rho)$ we can take

$$
T(\varepsilon ; \rho)(r, \phi)=\left(r^{\prime}, \phi^{\prime}\right)
$$

with

$$
\begin{aligned}
& r^{\prime}=r(1+\varepsilon \rho), \\
& \phi^{\prime}=\phi .
\end{aligned}
$$

Hence,

$$
\begin{aligned}
& T_{1}(\rho)(r, \phi)=(r \rho, 0), \\
& T_{n}(\rho)=0 \quad(n \geqslant 2) .
\end{aligned}
$$

We proceed with the calculation of $L_{n}(\rho)$.

The results are:

$$
\begin{aligned}
L_{0}(\rho)= & \mathscr{L} \\
L_{1}(\rho)= & -g \rho \mathscr{L}_{r}+r \rho h^{\prime}(r) D_{1} \\
& +h(r) \Sigma b_{p_{1} \ldots p_{\ell}}(\phi) A_{p_{1} \ldots p t}^{(1)}(\rho)+\Sigma c_{p_{1} \ldots p t}(\phi) B_{p_{1} \ldots p_{\ell}}^{(1)}(\rho),
\end{aligned}
$$

etc.

The $A_{p_{1} \ldots p t}^{(1)}(\rho)$ and $B_{p_{1} \ldots p_{t}}^{(1)}(\rho)$ are linear differential operators in $r$ and $\phi$ whose coefficients are homogeneous in $\rho$.

The next step is to determine the coefficients $U_{n}$ and $\lambda_{n}$ (see (1.11),(1.12)); $U_{1}$ will be a solution of

$$
\left(\mathscr{L}+\lambda_{0}\right) U_{1}=-\left(L_{1}+\lambda_{1}\right) U_{0}
$$

This problem has a solution if and only if

$$
\left(\left(L_{1}+\lambda_{1}\right) U_{0}, U_{0}\right)_{\omega_{0}}=0
$$

if we take $\left(U_{1}, U_{0}\right)=0$ the solution of $(2.12)^{(1)}$ is unique. The higher coefficients $U_{n}$ are determined by

$$
\left(\mathscr{L}+\lambda_{0}\right) U_{n}=-\sum_{i=1}^{n}\left(L_{i}+\lambda_{i}\right) U_{n-i}
$$

which has a unique solution perpendicular to $U_{0}$ if and only if

$$
\left(\sum_{i=1}^{n}\left(L_{i}+\lambda_{i}\right) U_{n-i}, U_{0}\right)=0 .
$$

By successively applying $(2.13)^{(k)},(2.12)^{(k)}(k=1,2, \ldots)$ we can determine all coefficients $U_{n}$ and $\lambda_{n}$. We shall soon need the explicit form of $\lambda_{1}$ and $U_{1}$ :

$$
\lambda_{1}=-g \lambda_{0} \gamma_{0} \frac{1}{\left(\Theta_{0}, 1\right)_{\Phi}},
$$


and

$$
U_{1}=\sum_{j \neq 0} \gamma_{j} \Theta_{j}(\phi) \sum_{i} \zeta_{i j}(r) u_{i j}
$$

with

$$
\begin{aligned}
u_{i j}= & \left\{\lambda_{0}-\mu_{i j}\left(R^{\prime}\right)\right\}^{-1}\left\{-g \lambda_{0}\left(\zeta_{0}, \zeta_{i j}\right)_{R^{\prime}}+\right. \\
& \left.-v_{j}\left(h r \frac{\mathrm{d}}{\mathrm{d} r} \zeta_{0}, \zeta_{i j}\right)_{R^{\prime}}-v^{\prime} j_{2}\left(r \frac{\mathrm{d}}{\mathrm{d} r} \zeta_{0}, \zeta_{i j}\right)_{R^{\prime}}\right\} .
\end{aligned}
$$

(we have omitted the superscript of $\zeta_{i, j}^{\left(R^{\prime}\right)} ; R^{\prime}=\left\{r: 0 \leqslant r<r_{0}(\lambda)\right\}$, see (2.6)).

We now want to write down $f_{1}(\rho)$. Let $G$ be Green's function belonging to $\mathscr{L}$, i.e. for every smooth $\chi$ :

$$
\left(\mathscr{L}^{-1} \chi\right)(s, \psi)=\int_{\Omega} G(s, \psi ; r, \phi) \chi(r, \phi) J(r, \phi) \mathrm{d} r \mathrm{~d} \phi,
$$

( $J$ is the Jacobian $r^{f} Q(\phi)$ ). We find:

$$
\begin{aligned}
f(\rho)(\psi)= & \left.\int_{\omega \infty} G(T(\rho(\psi))(s, \psi) ;(r, \phi)) u_{i n}(r, \phi) J(r, \phi) \mathrm{d} r \mathrm{~d} \phi\right|_{s=r_{0}} \\
= & \int_{\omega_{0}} G(T(\rho(\psi))(s, \psi) ; T(\rho(\phi))(r, \phi)) U(r, \phi) . \\
& \left.J(T(\rho(\phi))(r, \phi)) \cdot\left|T^{\prime}(\rho(\phi))\right| \mathrm{d} r \mathrm{~d} \phi\right|_{s=r_{0}}
\end{aligned}
$$

So

$$
\begin{aligned}
f_{1}(\rho) & =\left.\int_{\omega_{0}}\left(G_{s} s \rho(\psi) U_{0}+G_{r} r \rho(\phi) U_{0}+G U_{1}+(f+1) G U_{0} \rho(\phi)\right) \cdot r^{f} Q(\phi) \mathrm{d} r \mathrm{~d} \phi\right|_{s=r_{0}} . \\
& =\left.\int_{\omega_{0}}\left(G_{s} s \rho(\psi) U_{0}+G U_{1}-G r \rho(\phi) \frac{\partial U_{0}}{\partial r}\right) r^{f} Q(\phi) \mathrm{d} r \mathrm{~d} \phi\right|_{s=r_{0}} .
\end{aligned}
$$

Because of the restrictive assumptions which we have made for $\Omega$ and $\mathscr{L}$, we can now express Green's function $G$ in terms of the eigenfunctions of $\mathscr{L}$ on $\Omega$ :

Hence,

$$
G=-\sum_{i, j} \frac{E_{i j}(s, \psi) E_{i j}(r, \phi)}{\mu_{i j}} .
$$

$$
\begin{aligned}
f_{1}(\rho)= & -\sum_{i, j} \frac{\xi_{i j}^{\prime}\left(r_{0}\right) \Theta_{j}(\psi)}{\mu_{i j}} r_{0} \int \xi_{i j}(r) \Theta_{j}(\phi) \rho(\psi) \zeta_{0}(r) r^{f} Q(\phi) \mathrm{d} r \mathrm{~d} \phi \\
& -\sum_{i, j} \frac{\xi_{i j}\left(r_{0}\right) \Theta_{j}(\psi)}{\mu_{i j}} \int \xi_{i j}(r) \Theta_{j}(\phi)\left(\sum_{\substack{\ell m \\
\ell \geqslant 1}} \gamma_{i} \Theta_{l}(\phi) \zeta_{m \ell}(r) u_{m \ell}\right) r^{f} Q(\phi) \mathrm{d} r \mathrm{~d} \phi \\
& +\sum_{i, j} \frac{\xi_{i j}\left(r_{0}\right) \Theta_{j}(\psi)}{\mu_{i j}} \int \xi_{i j}(r) \Theta_{j}(\phi) r \rho(\phi) \frac{\mathrm{d}}{\mathrm{d} r} \zeta_{0}(r) r^{f} Q(\phi) \mathrm{d} r \mathrm{~d} \phi
\end{aligned}
$$


Therefore,

$$
f_{1}(\rho)=\sum_{j} \gamma_{j} \Theta_{j}(\psi) \mathscr{F}_{j}\left(\lambda_{0}\right)
$$

with

$$
\begin{aligned}
\mathscr{F}_{j}\left(\lambda_{0}\right) & =\sum_{i} \frac{-\xi_{i 0}^{\prime}\left(r_{0}\right)}{\mu_{i 0}} r_{0} \int_{0}^{r_{0}} \xi_{i 0}(r) \zeta_{0}(r) r^{f} \mathrm{~d} r \\
& +\sum_{i} \frac{-\xi_{i j}\left(r_{0}\right)}{\mu_{i j}} \int_{0}^{r_{0}} \xi_{i j}(r) \sum_{m} \zeta_{m j}(r) u_{m j} r^{f} \mathrm{~d} r \\
& +\sum_{i} \frac{\xi_{i j}\left(r_{0}\right)}{\mu_{i j}} \int_{0}^{r_{0}} \xi_{i j}(r) \frac{\mathrm{d}}{\mathrm{d} r} \zeta_{0}(r) r^{s+1} \mathrm{~d} r . \quad\left[r_{0}=\left(\frac{\mu_{0}}{\lambda_{0}}\right)\right]^{1 / g}
\end{aligned}
$$

Bifurcation will occur only if a $j$ and a $\lambda_{0}$ exist such that $F_{j}\left(\lambda_{0}\right)=0$.

\subsection{Computation of higher order coefficients; non-linear bifurcation analysis}

We suppose $\mathscr{F}_{n}\left(\lambda_{0}\right)=0$ and $\mathscr{F}_{p}\left(\lambda_{0}\right) \neq 0(p \neq n)$. Decomposing the perturbation shape function as in (1.20), we obtain

$$
\rho=\gamma_{n}(\varepsilon) \Theta_{n}+\psi(\varepsilon)
$$

where $\gamma_{n}$ plays the role of $\kappa$ in Section 1 and

$$
\psi(\varepsilon)=\sum_{p \neq n} \gamma_{p}(\varepsilon) \Theta_{p}(\phi)
$$

where

$$
\begin{gathered}
\gamma_{0}(\varepsilon) \mathscr{F}_{0}=\left(-\varepsilon f_{2}\left(\gamma_{0} \Theta_{0}+\gamma_{n} \Theta_{n}\right)+\ldots+b, \Theta_{0}\right)_{\Phi}+0\left(\varepsilon^{2}\right), \\
\gamma_{p}(\varepsilon)=-\varepsilon \mathscr{F}_{p}^{-1}\left(f_{2}\left(\gamma_{0} \Theta_{0}+\gamma_{n} \Theta_{n}\right), \Theta_{p}\right)_{\Phi}+O\left(\varepsilon^{2}\right),(p \neq 0, n) .
\end{gathered}
$$

We further elaborate the expression for $\gamma_{p}(p \neq 0, n)$. We find:

$$
\gamma_{p}=\mathscr{F}_{p}^{-1} Y_{p n} \gamma_{n}^{2} \varepsilon+O\left(\varepsilon^{2}\right)
$$

An explicit expression for $Y_{p n}$ can be found in [8]; the most striking property of $Y_{p n}$ is its proportionality to $\left(\Theta_{p}, \Theta_{n}^{2}\right) ;(2.24),(2.25)$ and (2.27) together correspond to (1.22).

The bifurcation equation (cf. (1.23)) can be written as follows

$$
\left(f_{2}\left(\gamma_{0}+\gamma_{n} \Theta_{n}+\varepsilon \sum_{p \neq 0, n} \mathscr{F}_{p}^{-1} Y_{p n} \gamma_{n}^{2} \Theta_{p}\right)+\varepsilon f_{3}\left(\gamma_{0}+\gamma_{n} \Theta_{n}\right), \Theta_{n}\right)+O\left(\varepsilon^{2}\right)=0
$$

This equation must he solved (analogous to Section 1) for $\gamma_{0}$ in terms of $\gamma_{n}$ and $\varepsilon$, so that we can obtain $b(\varepsilon)$ through (2.25). Working out (2.28) we expect an expression

$$
\Gamma_{n} \gamma_{0}^{2}+\Xi_{n} \gamma_{0} \gamma_{n}+Y_{n n} \gamma_{n}^{2}+O(\varepsilon)=0
$$

$Y_{n n}$ can be obtained from (2.27) with $p=n ; \Gamma_{n}=0$ and $\Xi_{n}$ is given by a complicated formula; for details see [8]. We assume that $\Xi_{n} \neq 0$.

If $Y_{n n} \neq 0,(2.29)$ is solved by

$$
\gamma_{0}=\frac{-Y_{n n}}{\Xi_{n}} \gamma_{n}+O(\varepsilon)
$$


Substituting this in (2.14) we find

$$
\lambda-\lambda_{0}=g \lambda_{0} \frac{Y_{n n}}{\Xi_{n}}\left(\Theta_{0}, 1\right)^{-1} \gamma_{n} \varepsilon+O\left(\varepsilon^{2}\right),
$$

which is the analogue of (1.24).

Hence,

$$
\varepsilon \rho=\left(\lambda-\lambda_{0}\right) \frac{\Xi_{n}}{Y_{n n} g \lambda_{0}}\left(\Theta_{0}, 1\right) \Theta_{n}(\phi)-\left(\lambda-\lambda_{0}\right) \frac{\left(\Theta_{0}, 1\right)}{g \lambda_{0}} \Theta_{0}+O\left(\lambda-\lambda_{0}\right)^{2},\left(\lambda \approx \lambda_{0}\right) .
$$

This solves our problem; we observe 'bilateral' bifurcation.

We now recall that $Y_{n n}$ is proportional to $\left(\Theta_{n}, \Theta_{n}^{2}\right)$. In many cases (see Section 2.5$)$ this factor vanishes due to symmetry properties. If that is the case then in order to solve our problem we must calculate the third order terms in (2.29), from (2.28). Including third order terms, we expect from (2.28):

$$
\Xi_{n} \gamma_{0} \gamma_{n}+M_{n} \gamma_{n}^{3} \varepsilon+N_{n} \gamma_{n}^{2} \gamma_{0} \varepsilon+Q_{n} \gamma_{n} \gamma_{0}^{2} \varepsilon+P_{n} \gamma_{0}^{3} \varepsilon+O\left(\varepsilon^{2}\right)=0
$$

The interested reader can find the explicit form of the coefficient $M_{n}$ in [8]; we suppose $M_{n} \neq 0$. The solution of (2.33) now will be

$$
\gamma_{0}=-\frac{M_{n}}{\Xi_{n}} \gamma_{n}^{2} \varepsilon+O\left(\varepsilon^{2}\right)
$$

Moreover,

$$
\lambda_{2}=\Lambda \gamma_{n}^{2}+O(\varepsilon)
$$

so

$$
\lambda=\lambda_{0}+g \lambda_{0} \frac{M_{n}}{\Xi_{n}}\left(\Theta_{0}, 1\right)^{-1} \gamma_{n}^{2} \varepsilon^{2}+\Lambda \gamma_{n}^{2} \varepsilon^{2}+O\left(\varepsilon^{3}\right)
$$

Hence,

$$
\varepsilon \rho(\phi)= \pm\left\{\left(\lambda-\lambda_{0}\right)\left(g \lambda_{0} \frac{M_{n}}{\Xi_{n}}\left(\Theta_{0}, 1\right)^{-1}+\Lambda\right)^{-1}\right\}^{1 / 2} \Theta_{n}(\phi)+O\left(\lambda-\lambda_{0}\right) .
$$

This defines a unilaterally bifurcating pair of solutions of (1.1-1.5).

\subsection{Explicit examples}

2.5.1. The cylinder in $\mathbf{R}^{3}$. We study the cylinder $\Omega=\left\{(r, \phi, z) \in \mathbf{R}^{3}: 0 \leqslant r<1 ; 0 \leqslant \phi<2 \pi\right\}$, and $\mathscr{L}=\Delta$. We consider functions $u$ with the properties (a) $u(z+2 \pi)=u(z)$ in order to obtain a discrete spectrum for $\mathscr{L}$; (b) $\partial u / \partial \phi=0$ to simplify calculations, on the other hand we may miss bifurcating solutions which do not have this symmetry property. The eigenfunctions $\xi$ of $\mathscr{L}$ on $\Omega$, with constraints (a), (b) are

$$
\xi_{m k}=J_{0}\left(\ell_{m} r\right) e^{i k z} \mathscr{B}_{m k}
$$

with eigenvalues

$$
\mu_{m k}=\ell_{m}^{2}+k^{2}
$$


where $\ell_{m}$ is the $m^{\text {th }}$ zero of $J_{0}$ (Bessel function of the first kind and the zero order) and $\mathscr{B}_{m k}$ is a normalization constant. The normalized eigenfunctions $\zeta$ of $\mathscr{L}$ on $\omega=\left\{(r, \phi, z): 0 \leqslant r<r_{0}\right.$; $0 \leqslant \phi<2 \pi\}$ are

$$
\zeta_{m k}=J_{0}\left(\ell_{m} \frac{r}{r_{0}}\right) e^{i k z} \frac{\mathscr{B}_{m k}}{r_{0}} .
$$

The basic branch of solutions is given by the domain

$$
\omega(\lambda)=\left\{(r, \phi, z): 0 \leqslant r<r_{0} ; 0 \leqslant \phi<2 \pi\right\} ; r_{0}=\frac{\ell_{0}}{\sqrt{\lambda}}
$$

A numerical treatment of (2.22) gives

$$
\begin{aligned}
& 1^{\text {st }} \text { bifurcation point: } r_{0}{ }^{(1)}=0.92254, \\
& 2^{\text {nd }} \text { bifurcation point: } r_{0}{ }^{(2)}=0.75000, \\
& 10^{\text {th }} \text { bifurcation point: } r_{0}{ }^{(10)}=0.17527
\end{aligned}
$$

The basic branch corresponds to a central, $\phi$ - and $z$-independent column. The solution bifurcating from the $n^{\text {th }}$ bifurcating point corresponds in first approximation to a central, $\phi$ independent column with $2 \pi / n$-periodic deformation of the boundary (see Fig. 1).
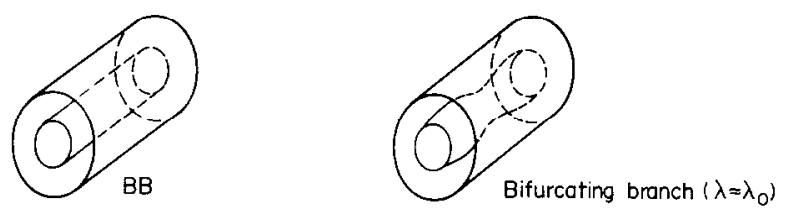

Fig. 1.

There is a small complication with respect to the previous analysis (Sections 2.1-2.4), because the bifurcation points are degenerate $\left(\mathscr{F}_{n}\right.$ and $\mathscr{F}_{-n}$ vanish simultaneously). This degeneration leads to a bifurcation equation (cf. (2.34)):

$$
\gamma_{0}=-\frac{M_{n}}{\Xi_{n}} \gamma_{n} \gamma_{-n} \varepsilon+O\left(\varepsilon^{2}\right)
$$

and (2.35) becomes

$$
\lambda-\lambda_{0}=\gamma_{n} \gamma_{-n} \varepsilon^{2}\left(g \lambda_{0} \frac{M_{n}}{\Xi_{n}}\left(\Theta_{0}, 1\right)^{-1}+\Lambda\right)+O\left(\varepsilon^{3}\right),
$$

where $\gamma_{ \pm n}$ is the (complex) coefficient of $\mathrm{e}^{ \pm i n z}$. Because $\gamma_{-n}=\bar{\gamma}_{n}$, only $\left|\gamma_{n}\right|^{2}$ is determined and the phase of $\gamma_{n}$ is arbitrary. This gives rise to a one-parameter set of bifurcating solutions: for every $u(r, \phi, z), 0<\alpha<2 \pi, u(r, \phi, z+\alpha)$ also is a solution.

For the deformation of the boundary corresponding to the first bifurcating solution we find after much computational work (the calculation of $M_{n}, \Xi_{n}$ and $\lambda$ ):

$$
\gamma(z)=r_{0}+r_{0} \sqrt{\lambda-\lambda_{0}} \cdot 0 \cdot 0772 \cos (z+\alpha)+O\left(\lambda-\lambda_{0}\right)
$$


2.5.2. The strip in $\mathbf{R}^{2}$. We consider the strip $\Omega=\left\{(x, y) \in \mathbf{R}^{2}: 0<x<1\right\}$ and functions $u$ with $u(y+2 \pi)=u(y)$; for $\mathscr{L}$ we take again $\mathscr{L}=\Delta$. The normalized eigenfunctions are

$$
\xi_{m n}=\frac{1}{\sqrt{\pi}} \mathrm{e}^{i m y} \sin \pi n x
$$

with eigenvalues $m^{2}+\pi^{2} n^{2}$. For $\lambda \geqslant \pi^{2}$ the basic branch is given by

$$
\omega_{0}=\left\{(x, y) \in \mathbf{R}^{2}: \frac{1-r_{0}(\lambda)}{2}<x<\frac{1+r_{0}(\lambda)}{2}\right\}, \quad \text { with } r_{0}(\lambda)=\pi \lambda^{-1 / 2} \text {. }
$$

A perturbation of the two components of the free boundary is now represented by

$$
\begin{aligned}
& x_{u}^{\prime}=\frac{1+r_{0}}{2}+r_{0} \gamma(y), \\
& x_{\ell}^{\prime}=\frac{1-r_{0}}{2}-r_{0} \beta(y) .
\end{aligned}
$$

where $x_{u}^{\prime}$ denotes the upper boundary and $x_{\ell}^{\prime}$ the lower one. The transformation that maps the perturbed domain to the basic one is given by

$$
x^{\prime}=x(1+\gamma+\beta)+\frac{r_{0}-1}{2} \gamma-\frac{1+r_{0}}{2} \beta
$$

Proceeding now as in Section 1, we must define functions $f_{u}$ and $f_{\ell}$ (cf. (1.16)), for $r=\left(1 \pm r_{0}\right) / 2$, respectively, which have to satisfy

$$
f_{u}\left(\frac{1+r_{0}}{2}\right)=f\left(\frac{1-r_{0}}{2}\right)=\text { constant }
$$

The linear approximation of these equations leads to

$$
\left(\begin{array}{lr}
\mathscr{F}_{n}(\lambda)-G_{n}(\lambda) \\
\mathscr{F}_{n}(\lambda) & G_{n}(\lambda)
\end{array}\right)\left(\begin{array}{l}
\gamma_{n}+\beta_{n} \\
\gamma_{n}-\beta_{n}
\end{array}\right)=\left(\begin{array}{l}
0 \\
0
\end{array}\right), \quad(n \neq 0)
$$

where the $\mathscr{F}_{n}$ and $G_{n}$ are real functions; $G_{n}(\lambda)$ appears to have no zeroes. Non-invertibility arises for $\mathscr{F}_{n}(\lambda)=0$; we expect a bifurcating branch which is in its first approximation proportional to $\gamma=\gamma \mathrm{e}^{i n y}, \beta=\gamma_{n} \mathrm{e}^{i n y}$,

We list some of the calculated bifurcation points:

$$
\begin{aligned}
& n=1: \lambda \approx 10.87, \\
& n=2: \lambda \approx 13.96, \\
& n=3: \lambda \approx 19.55 .
\end{aligned}
$$

\section{GENERAL THEORY}

\subsection{Preliminaries}

In this section we shall give a more rigorous treatment of the free boundary eigenvalue problem. For completeness we restate the problem.

Let $\Omega \subset \mathbf{R}^{n}$ be an open connected set with a smooth boundary $\partial \Omega$, and let $\lambda$ be a real number. 
We search for a domain $\omega$ with $\bar{\omega} \subset \Omega$, and a function $u$ defined on $\Omega$ such that

$$
\begin{aligned}
\mathscr{L} u+\lambda u & =0 \text { in } \omega, \\
\mathscr{L} u & =0 \text { in } \Omega \backslash \bar{\omega}, \\
u & =0 \text { on } \partial \omega, \\
u(x) & >0 \Leftrightarrow x \in \omega, \\
u & =-1 \text { on } \partial \Omega,
\end{aligned}
$$

while $\omega$ and $u$ satisfy the smoothness properties

$$
\begin{aligned}
& u \in \mathscr{D}, \\
& \omega \in G_{p} .
\end{aligned}
$$

A characterization of $\mathscr{D}$ and $G_{p}$ will be given shortly. Here $\mathscr{L}$ is a uniformly elliptic operator of the form

$$
\mathscr{L}=\sum_{i, j=1}^{n} \frac{\partial}{\partial x_{i}} \alpha_{i j}(x) \frac{\partial}{\partial x_{j}}
$$

with smooth coefficients $\alpha_{i j} ; \alpha_{i j}=\alpha_{j i}$. There is a Green's function $G$ for the operator $\mathscr{L}$, i.e. the problem

$$
\begin{aligned}
\mathscr{L} u & =f \text { in } \Omega, \\
u & =0 \text { on } \partial \Omega
\end{aligned}
$$

is solved by

$$
u(x)=\int_{\Omega} G(x ; \xi) f(\xi) \mathrm{d} \xi
$$

provided $f$ is sufficiently smooth.

Remarks. On function spaces and norms.

Let $p$ be a real positive number and $X$ a compact subset of $\mathbf{R}^{n}$ with induced norm $\|\cdot\|$.

(1) $[p]$ is the largest integer, smaller than or equal to $p$.

(2) A real function $f$ on $X$ is $\alpha$ Hölder continuous $(0<\alpha<1)$ if the semi-norm

$$
\|f\|_{\alpha}=\sup _{x, y \in X} \frac{|f(x)-f(y)|}{\|x-y\|^{\alpha}}
$$

is finite.

(3) $C^{p}(X)$ is the set of $[p]$ times differentiable functions on $X$, with $p-[p]$ Hölder continuous $p^{\text {th }}$ order derivatives. On $C^{p}(X)$ we define the norm

$$
\|f\|_{p}=\sum_{\substack{0 \leqslant i ! i \leqslant p \\ l \in N}} \sup _{x \in X}\left|f^{(i)}(x)\right|+\sum_{\mid \ell=[p]}\left\|f^{(\theta)}\right\|_{p-[p]},
$$

(if $p$ is an integer we omit the last term).

If ambiguity may arise, we also write $\|f\|_{C_{(X)} \text {. }}$. 
(4) If $m$ is an integer, we define $C^{m-}(X)=\bigcap_{m-1<p<m} C^{p}(X)$.

(5) $C^{p}\left(X, \mathbf{R}^{n}\right)$ is the set of all $n$-vectorfunctions on $X$ with each component in $C^{p}(X)$, equipped with $\|\cdot\|_{p, \mathbf{R}^{n}}=$ the sum of the $\|\cdot\|_{p}$ norms on the components.

(6) $H^{k, t}(X)$ is the space of functions of which all $k^{\text {th }}$ order distributional derivatives are in $L_{t}(X)$.

(7) In some applications (see Section 2) it may happen that the domain $\Omega$ itself is unbounded but can be generated by periodic continuation from a bounded fundamental cell $\tilde{\Omega} \subset \Omega$. In that case we can study our problem on $\tilde{\Omega}$; for details of such an analysis (for the Navier-Stokes problem), see e.g. Iooss [12].

For the space $\mathscr{D}$ we now require

$$
\mathscr{D}=C^{2}(\omega) \cap C^{2}(\Omega \backslash \bar{\omega}) \cap C^{1}(\Omega)
$$

Next, we define the set of admissible domains and boundaries; $D_{n}$ is the open unit ball in $\mathbf{R}^{n}$.

Definition 1. $\omega$ is an admissible domain if there exist a neighbourhood $W$ of $D_{n}$ and a 1-1 mapping $T: W \rightarrow \mathbf{R}^{n}$ with $T \in C^{p}\left(W, \mathbf{R}^{n}\right)$ and $T^{-1} \in C^{p}\left(T W, \mathbf{R}^{n}\right)$, such that $\omega=T\left(D_{n}\right)$ and $\partial \omega=T\left(\partial D_{n}\right)$. The set of admissible domains, for any fixed $p$, will be denoted by $G_{p}$.

We intend to study domains $\omega$ which are 'close' to a certain $\omega_{0}$; therefore we define a topology (even a metric) on $G_{p}$.

Definition 2. The distance between two domains $\omega_{1}, \omega_{2} \in G_{p}$ is $\varepsilon$ if

$$
\inf _{\substack{T_{1} \in \bar{T}_{1} \\ i_{2} \in \sigma_{2}}}\left\|T_{1}-T_{2}\right\|_{p, \mathbf{R}^{n}}=\varepsilon
$$

Here $\mathscr{T}_{i}$ is the set of all $T_{i}$ belonging to $\omega_{i}$ in the sense of Definition 1.

For further analysis we want to represent the admissible domains by their boundaries. So we formulate Definition 3.

Definition 3. Let $\omega \in G_{p}$ and let $T$ be as in Definition 1. The restriction of $T$ to $\partial D_{n}$ is denoted by $g$. The set of all such mappings $g$ will be denoted by $\mathscr{B}_{p}$.

We observe $\mathscr{B}_{p} \subset C^{p}\left(\partial D_{n} ; \mathbf{R}^{n}\right)$; this enables us to define a metric on $\mathscr{B}_{p^{*}}$

Definition $4 . \mathscr{B}_{p}$ is provided with the topology induced by $\|\cdot\|_{p, \mathbf{R}^{n}}$.

Lemma 1. Let $p \geqslant 1, g_{0} \in \mathscr{B}_{p}$ and $\omega_{0} \in G_{p}$, with $g_{0}\left(\partial D_{n}\right)=\partial \omega_{0}$ cf. Definition 3. Then:

(i) $g \in C^{p}\left(\partial D_{n} ; \mathbf{R}^{n}\right)$ and $\left\|g-g_{0}\right\|_{C^{1}}$ small implies $g \in \mathscr{B}_{p}$.

Let $\omega \in G_{p}$ and $g \in \mathscr{B}_{p}$ with $g\left(\partial D_{n}\right)=\partial \omega$, cf. Definition 3;

(ii) $g \rightarrow g_{0}$ in $\mathscr{B}_{p}$ implies $\omega \rightarrow \omega_{0}$ in $G_{p}$;

(iii) $\omega \rightarrow \omega_{0}$ in $G_{p}$ implies there are $g$ in $\mathscr{B}_{p}, g\left(\partial D_{n}\right)=\partial \omega$, such that $g \rightarrow g_{0}$.

Proof of Lemma 1 .

(i) Let $g_{0} \in \mathscr{B}_{p}, T_{0}$ a 1-1 $C^{p}$ map from $W_{0}$ onto $V_{0}=T_{0} W_{0}$ with $\partial D_{n}$-restriction $g_{0}$, as meant in Definitions 1, 3. Because $\overline{\omega_{0}} \subset V_{0}$ we have $g\left(\partial D_{n}\right) \subset V_{0}$, so $T_{0}^{-1} g$ is defined as a 1-1 mapping from $\partial D_{n}$ to $W_{0}$. 
We introduce polar coordinates $\left(r, \phi, \ldots, \phi_{n-1}\right)$ in $W_{0}$ and we define mappings $\pi_{\phi}$ and $\pi_{r}$ by $\pi_{\phi}(r, \phi)=\phi$ and $\pi_{r}(r, \phi)=r$.

Let $S(g, \cdot)$ be given by $S(g, \phi)=\pi_{\phi} T_{0}^{-1} g(\phi)$. $S$ is differentiable with respect to $\phi$ and the linear approximation converges to the identity if $\left\|g-g_{0}\right\|_{C^{1}} \rightarrow 0$. So for $\left\|g-g_{0}\right\|_{C^{1}}$ small enough, $S\left(g,,^{\circ}\right)$ is invertible and we denote its inverse by $P(g, \cdot)$.

Let $R(g, \phi)=\pi_{r}\left(T_{0}^{-1} g(\phi)\right)$ and define on $Z_{0} \subset W_{0} T_{g}$ by $T_{g}(r, \phi)=T_{0}(r R(g, \phi), S(g, \phi))$. Clearly, $T_{g}$ is in $C^{p}\left(Z_{0}, \mathbf{R}_{n}\right)$. Moreover, $T_{g}$ is invertible and its inverse is given by

$$
T_{g}^{-1}(x)=\left(\frac{\pi_{r}\left(T_{0}^{-1} x\right)}{R\left(g, P\left(g, \pi_{\phi} T_{0}^{-1} x\right)\right)}, P\left(g, \pi_{\phi} T_{0}^{-1} x\right)\right) ; \quad\left(x \in T_{g} Z_{0}\right) .
$$

$T_{g}^{-1}$ is in $C^{p}\left(T Z_{0}, Z_{0}\right)$, so $T_{g}\left(D_{n}\right) \in G_{p}$.

On $\partial D_{n}, T_{g}$ coincides with $g$.

(ii) Follows immediately from the choice of $T_{g}$;

(iii) Is trivial.

A consequence of the proof of this lemma is that for $p \geqslant 1, \mathscr{B}_{p}$ is an open subset of the Banach space $C^{p}\left(\partial D_{n} ; \mathbf{R}^{n}\right)$.

The space $\mathscr{B}_{p}$ is not yet suitable for a formulation of the bifurcation problem; therefore we perform one more reduction.

Let $g_{0} \in \mathscr{B}_{p}$ and let $T_{0}$ be a corresponding mapping (cf. Definition 3). Let 1 be the constant $=1$ function on $\partial D_{n}\left(1 \in C^{p}\left(\partial D_{n}\right)\right)$ and take an element $\gamma$ of $C^{p}\left(\partial D_{n}\right),\|\cdot\|_{p}$ - close to 1 . If we now introduce polar coordinates $(r, \phi)$ in a neighbourhood of $D_{n}$, then $\sigma(\gamma)$, defined by $\sigma(\gamma)(\phi)=T_{0}(\gamma(\phi), \phi)$ is an element of $\mathscr{B}_{p}$, close to $g_{0}$ in the $\mathscr{B}_{p}$-topology. $\sigma$ has a kind of pseudo-inverse, as is demonstrated by the following lemma.

Lemma 2. Let $g_{0} \in \mathscr{B}_{p}, p \geqslant 1$ and let $1: \partial D_{n} \rightarrow \mathbf{R}$ be the constant $=1$ function. Then there exist a neighbourhood $U \subset \mathscr{B}_{p}$ of $g_{0}$, a neighbourhood $V \subset C^{p}\left(\partial D_{n}\right)$ of 1 , a $C^{p}$ map $\tau$ from $U$ onto $V$, and a 1-1 $C^{p}$ map $\sigma$ from $V$ into $U$ with $\tau \odot \sigma=$ the identity on $V$. Furthermore, for every $g \in U$, $\sigma(\tau(g))$ represents the same boundary as $g$.

Proof. Let $U^{1} \subset \mathscr{B}_{p}$ be a small neighbourhood of $g_{0}$. We first define $\tau$ on $U^{1} ;$ let $S, R, P$ be as defined in the proof of Lemma 1.

Now take $\tau(g)=\gamma$ with $\gamma(\phi)=R(g, P(g, \phi))$. Clearly, $\gamma \in C^{p}\left(\partial D_{n}, \mathbf{R}\right)$. Next, we define $\sigma$ on a neighbourhood of 1 in $C^{p}\left(\partial D_{n}, \mathbf{R}\right)$ by $\sigma(\gamma)=g$ with $g(\phi)=T_{0}(\gamma(\phi), \phi)$. Because $T_{0}$ is $C^{p}$, there is a $C^{p}$-neighbourhood $V$ of 1 such that $\sigma(V)-U^{1}$. Choose $U=\tau^{-1}(V) \cap U^{1}$. The rest of the properties of $\sigma$ and $\tau$ are obvious.

COROllary 1. To Lemmas 1 and 2: all small perturbations in $G_{p}$ of $\omega_{0} \in G_{p}$ are covered if we study a neighbourhood of $1 \in C^{p}\left(\partial D_{n}\right)$.

From the proofs of Lemmas 1 and 2 we immediately find the following.

COROllary 2 . To Lemmas 1 and $2:$ let $p \geqslant 1$ and $k \leqslant p$ a natural number. A family $\omega(\varepsilon)$, for which the $T(\varepsilon)$ (cf. Definition 1 ) depend $k$-differentiably on $\varepsilon$, corresponds through a family $g(\varepsilon) \subset$ $C^{p}\left(\partial D_{n} ; \mathbf{R}^{n}\right)$ to a family $\gamma(\varepsilon) \subset C^{p}\left(\partial D_{n}\right)$, depending $k$-differentiably on $\varepsilon$, and vice versa. 
We now use a result of Berestycki-Brézis [9], which states that a basic branch

$$
\mathscr{B} \mathscr{B}=\{\omega, u, \lambda\} \subset G_{p_{0}} \times \mathscr{D} \times \mathbf{R},
$$

of solutions of (3.1-3.7) exists; let $p \leqslant p_{0}$ and $k \in \mathbf{N}, k \leqslant p$.

Definition 3. $\lambda_{0}$ is a bifurcation value for $\mathscr{B} \mathscr{B}$ and $\left\{\omega_{0}, u_{0}, \lambda_{0}\right\}$ is a bifurcation point of type $(p, k)$, if (i) there is a solution branch $\tilde{\mathscr{B}}=\{\tilde{\omega}, \tilde{u}, \lambda\} \subset G_{p} \times \mathscr{D} \times \mathbf{R}$ such that $\tilde{\mathscr{B}} \cap \mathscr{B}=\left\{\omega_{0}, u_{0}, \lambda_{0}\right\}$,

(ii) the boundary functions $\tilde{\gamma}$ corresponding to the $\tilde{\alpha}$ can be parametrized as a $C^{k}$ function of the

1 dimensional variable $\varepsilon$.

In the rest of this section we shall characterize the possible bifurcation values $\lambda_{0}$ and the bifurcating branches $\tilde{\mathscr{B}}$. So we start with a $\lambda_{0}$ (at this moment arbitrary) and an arbitrary $C^{k}$ family $\tilde{\gamma}(\varepsilon) \subset C^{p}\left(\partial D_{n}\right)$ near 1 , and we try to find out whether $\lambda_{0}$ is a bifurcation value and $\tilde{\gamma}(\varepsilon)$ represents the corresponding branch.

\subsection{Solution in the inner domain $(\tilde{\omega})$}

Suppose $\lambda_{0}$ is a bifurcation value and $\tilde{\gamma}(\varepsilon)=1+\varepsilon \rho, \rho \in C^{p}\left(\partial D_{n}\right)$. We first consider the eigenvalue problem on $\tilde{\omega}(\varepsilon)$, i.e. the domain belonging to $\tilde{\gamma}(\varepsilon)$ :

$$
\begin{aligned}
\mathscr{L} u_{i n}+\lambda u_{\text {in }} & =0 \text { in } \tilde{\omega}(\varepsilon), \\
u_{\text {in }} & =0 \text { on } \tilde{\partial} \tilde{\omega}(\varepsilon), \\
u_{\text {in }} & >0 \text { in } \tilde{\omega}(\varepsilon) .
\end{aligned}
$$

Of course, the eigenfunction $u_{i n}$ and the eigenvalue $\lambda$ of this problem depend on $\varepsilon$ and $\rho$, so we shall write $u_{\text {in }}(\varepsilon, \rho, x)(x \in \tilde{\omega}(\varepsilon))$ and $\lambda(\varepsilon, \rho)$.

Because $u_{i n}$ is the first eigenfunction of $\mathscr{L}$ on $\tilde{\omega}(\varepsilon)$, it has to minimize

$$
\int_{\tilde{\omega}(\varepsilon)} \sum_{i, j} \alpha_{i j} \frac{\partial}{\partial x_{i}} u \frac{\partial}{\partial x_{j}} u \mathrm{~d} x,
$$

(defined on $H_{0}^{1}(\tilde{\omega}(\varepsilon))$ ), under the condition

$$
\int_{\tilde{\omega}(\varepsilon)} u^{2} \mathrm{~d} x=1
$$

If we now apply the following transformation of coordinates:

$$
y=T_{\sigma(\hat{y}(u))}^{-1}(x)
$$

where $T_{\sigma(\tilde{\gamma}(\varepsilon))}$ is defined for $y$ (expressed in polar coordinates $y=(r, \phi)$ )

$$
T_{\sigma(\hat{\gamma}(\varepsilon))}(y)=T_{0}(r(1+\varepsilon \rho(\phi)), \phi) .
$$

(3.19) maps $\tilde{\omega}(\varepsilon) 1-1 C^{p}$ onto $D_{n}$ and if we define

$$
U(\varepsilon, \rho, y)=u_{i n}(\varepsilon, \rho, x)
$$

we find that $U$ has to minimize

$$
\int_{D_{n}} \sum_{i, j} c_{i j} \frac{\partial v}{\partial y_{i}} \frac{\partial v}{\partial y_{j}} J \mathrm{~d} y
$$


(defined on $H_{0}^{1}\left(D_{n}\right)$ ), under the condition

$$
\int_{D_{n}} v^{2} J \mathrm{~d} y=1
$$

where

$$
c_{i j}(y)=\sum_{k, \ell} \alpha_{k_{1}}(x) \frac{\partial\left[T_{\sigma(\hat{\gamma}(\varepsilon))}^{-1}(x)\right]_{j}}{\partial x_{k}} \cdot \frac{\partial\left[T_{\sigma(\gamma(\varepsilon))}^{-1}(x)\right]_{i}}{\partial x_{t}} \mid x=T_{\sigma(\hat{\gamma}(\varepsilon))} y,
$$

and $J(\varepsilon, \rho, y)$ is the Jacobian of the transformation:

$$
J=\operatorname{det}\left(\frac{\partial\left[T_{\sigma(\tilde{\gamma}(\varepsilon))}(y)\right]_{i}}{\partial y_{j}}\right)
$$

We now devote some attention to $T_{\sigma(\hat{y}(r))^{\circ}}^{-1}$. If $T_{\sigma(\tilde{y}(\varepsilon))}^{-1}(x)=y=(r, \phi)$ and $\pi_{r}\left(T_{0}^{-1}(x)\right)$ is the $r$ coordinate of $T_{0}^{-1} x$ and $\pi_{\phi}\left(T_{0}^{-1} x\right)$ is its $\phi$-coordinate, then

$$
r=\frac{\pi_{r}\left(T_{0}^{-1}(x)\right)}{1+\varepsilon \rho\left(\pi_{\phi}\left(T_{0}^{-1}(x)\right)\right)}, \quad \phi=\pi_{\phi}\left(T_{0}^{-1}(x)\right),
$$

so because $T_{0}^{-1} \in C^{p_{0}}, \rho \in C^{p}$ and $p \leqslant p_{0}, y$ depends $C^{p}$ on $x$ and analytically on $\varepsilon$.

It follows that the coefficients $b_{i j}$ and $J$ are $C^{p-1}$ functions of $y$, analytical in $\varepsilon$, so with Kato [13], chap. VII, section 6 , we find (i) $\lambda$ is an analytic function of $\varepsilon$, (ii) $U \in H_{0}^{1}\left(D_{n}\right)$ is an analytic function of $\varepsilon$.

We shall derive a better $y$-regularity for $u_{i n}$. Because $u_{i n} \in H_{0}^{1}(\tilde{\omega}(\varepsilon)), \partial \tilde{\omega}(\varepsilon)$ is $C^{p}$ and $u_{i n}$ satisfies (3.14), (3.15) we find by repeatedly applying Friedman [14] Th. 17.2: $u_{i n} \in H^{[p]}(\tilde{\omega}(\varepsilon)$ ), provided $[p] \geqslant 2$. If, moreover, $[p] \geqslant n / 2$ we have $u_{i n} \in C^{0}(\tilde{\omega}(\varepsilon))$ and with Agmon et al. [15] we obtain $u_{i n} \in C^{p} \overline{(\tilde{\omega}(\varepsilon))}$, provided $p \notin \mathbf{N}$. Because $T_{\sigma(\hat{\gamma}(\varepsilon))}^{-1}$ is $C^{p}, U \in C^{r}\left(\overline{\left.D_{n}\right)}\right.$ also.

We now investigate the $\varepsilon$-dependence of $u_{i n}$. Analyticity with respect to $\varepsilon$ leads to

$$
\begin{gathered}
\left(y \in D_{n}\right), u(\varepsilon, \rho, y)=\sum_{m \geqslant 0} \varepsilon^{m} U_{m}(\rho, y), \\
\lambda(\varepsilon, \rho)=\sum_{m \geqslant 0} \varepsilon^{m} \lambda_{m}(\rho) .
\end{gathered}
$$

We shall derive some results for $U_{m}$ and $\lambda_{m}$.

Lemma 4. Let $\tilde{\gamma}(\varepsilon)=1+\varepsilon \rho, \rho \in C^{p}\left(\partial D_{n} ; \mathbf{R}\right)$, define a perturbation of the boundary of the suggested bifurcation point $\left\{\omega_{0}, u_{0}, \lambda_{0}\right\}$. Then if $p \leqslant p_{0},[p] \geqslant \max (n / 2,2), p \notin \mathbf{N}$, there is a solution of problem $(3.14,3.15,3.16)$ with properties $(3.27,3.28,3.39)$ where the $U_{m}$ and $\lambda_{m}$ satisfy

$$
\begin{gathered}
\left\|U_{m}\right\|_{p} \leqslant\|\rho\|_{p}^{m} . C, \\
\left|\lambda_{m}\right| \leqslant\|\rho\|_{p}^{m} . C .
\end{gathered}
$$

Proof. The equation

$$
\mathscr{L} u_{i n}+\lambda u_{i n}=0
$$

becomes after transformation (3.19):

$$
L U+\lambda U=0
$$


where

$$
L=\sum_{i, j} \sum_{k, \ell} \frac{\partial\left[T_{\sigma(\dot{\gamma}(\varepsilon))}^{-1}(x)\right]_{k}}{\partial x_{i}} \frac{\partial}{\partial y_{k}}\left\{\alpha_{i j}(x) \frac{\partial\left[T_{\sigma(\tilde{y}(\varepsilon))}^{-1}(x)\right]_{\ell}}{\partial x_{j}} \frac{\partial}{\partial y_{l}}\right\},
$$

which can be written in a Taylor expansion

$$
L=\sum_{m \geqslant 0} \varepsilon^{m} L_{m}(\rho)
$$

with

$$
L_{0}=\mathscr{L} \circlearrowleft T_{0}^{-1}
$$

and, in general,

$$
L_{m}=\sum_{i, j} \mathscr{A}_{i j m} \frac{\partial}{\partial y_{i}} \frac{\partial}{\partial y_{j}}+\sum_{i} \mathscr{B}_{i m} \frac{\partial}{\partial y_{i}}
$$

here $\mathscr{A}_{i j m} \in C^{p-1} \overline{\left(D_{n}\right)}$ and $\mathscr{B}_{i m} \in C^{p-2} \overline{\left(D_{n}\right)} \cdot \mathscr{A}_{i j m}$ and $\mathscr{B}_{i m}$ are $m$-homogeneous in $\rho$ and

$$
\left\|\mathscr{A}_{i j m}\right\|_{p-1} \leqslant \alpha_{i j m}\|\rho\|_{p}^{m}, \quad\left\|\mathscr{B}_{i m}\right\|_{p-2} \leqslant \beta_{i m}\|\rho\|_{p}^{m}
$$

( $\alpha_{i j m}$ and $\beta_{i m}$ are positive real constants). We have

$$
U_{0}(\rho, y)=u_{0}\left(T_{0} y\right), \quad\left(y \in D_{n}\right)
$$

and the $U_{m}(m>0)$ have to satisfy

$$
\begin{aligned}
\left(L_{0}+\lambda_{0}\right) U_{m} & =-\sum_{i=1}^{m}\left(L_{i}+\lambda_{i}\right) U_{i-m} \text { in } D_{n}, \\
U_{m} & =0 \text { on } \partial D_{n},
\end{aligned}
$$

which is solvable if and only if

$$
\mathscr{P}\left(\sum_{i=1}^{m}\left(L_{i}+\lambda_{i}\right) \quad U_{i-m}\right)=0
$$

where $\mathscr{P}$ is the projection onto $U_{0}$ (we have applied the Fredholm alternative to $L_{0}$ ). Now by applying $(3.40,1),(3.38,1),(3.40,2),(3.38,2)$, etc. we can determine $\lambda_{m}$ and $U_{m}$ alternately $(m>0)$. We find $U_{m} \in C^{p}\left(\overline{D_{n}}\right) ; \lambda_{m}$ and $U_{m}$ are $m$-homogeneous in $\rho$, and (3.29), (3.30) follow from (3.36), (3.38) and (3.40).

3.3 Solution in the outer domain and the interface condition

Now that we have determined $u_{i n}$ inside $\tilde{\omega}$, we can turn to the study of $\Omega \backslash \tilde{\omega}$.

Wc definc $u_{\text {pre }}$ as the classical solution of

$$
\begin{aligned}
\mathscr{L} u_{\mathrm{pre}}+\lambda u_{i n,+} & =0 \text { in } \Omega \\
u_{\mathrm{pre}} & =0 \text { on } \partial \Omega
\end{aligned}
$$

where

$$
\begin{aligned}
u_{\text {in },+} & =u_{\text {in }} \text { in } \tilde{\omega}(\varepsilon), \\
& =0 \text { in } \Omega \backslash \tilde{\omega}(\varepsilon) .
\end{aligned}
$$


Because $u_{i n} \in C^{p}(\overline{\tilde{\omega}(\varepsilon)})$ we find $u_{i n,+} \in C^{1-}(\bar{\Omega})$, provided $p \geqslant 1$. From Agmon et al. [15] Th. 7.3 we find $u_{\text {pre }} \in C^{3-}(\bar{\Omega}) \cup C^{p}(\bar{\Omega})$. On $W_{0} \backslash D_{n}\left(W_{0}\right.$ corresponds to $\omega_{0}$ as in Definition 1$)$ we define

$$
\mathscr{F}(\varepsilon, \rho, y)=-(\lambda(\varepsilon, \rho))^{-1} u_{\mathrm{pre}}\left(T_{\sigma(\hat{\gamma} \gamma(\xi))}(y)\right) \text {. }
$$

Evidently, $\mathscr{F}(\varepsilon, \rho, \cdot) \in C^{3-}\left(W_{0} \backslash D_{n}\right) \cup C^{p}\left(W_{0} \backslash D_{n}\right)$. Let $f$ be the restriction of $\mathscr{F}$ to $\partial D_{n} ; f(\varepsilon, \rho, \cdot) \in$ $C^{3-}\left(\partial D_{n}\right) \cup C^{p}\left(\partial D_{n}\right)$. If now, for some $\rho, f(\varepsilon, \rho, \cdot)=1 \cdot C$ where 1 is the constant 1 function on $\partial D_{n}$ and $C$ is a constant which can depend on $\varepsilon$, then in $\tilde{\omega} u_{\text {pre }}+C \lambda$ equals $u_{i n}$, hence,

$$
\tilde{u}=-\frac{u_{\mathrm{pre}}+C \lambda}{C \lambda}
$$

meets all conditions (3.1-3.7) and our problem is solved.

So the question of $\lambda_{0}$ being a bifurcation value and $\tilde{\gamma}(\varepsilon)=1+\varepsilon \rho$ representing a bifurcation branch simply amounts to solving the problem: is there a $\rho \in C^{p}\left(\partial D_{n} ; \mathbf{R}\right)$ such that $f(\varepsilon, \rho, \cdot)$ is a constant function on $\partial D_{n}$ ?

\subsubsection{Properties of the operator $\mathrm{f}$.}

(i) $y$-regularity of $f$.

In the above we found $f(\varepsilon, \rho, \cdot) \in C^{\prime}\left(\partial D_{n} ; \mathbf{R}\right)$ with $\ell<3$ and $\ell \leqslant p$. To obtain a better result for the regularity of $f$ we prove the following lemma.

LEMmA 5. Let $p$ and $q$ be non-integer real numbers with $p, q>2$, and let $I$. be a differential operator of the form

$$
L=\sum_{i, j} \mathscr{A}_{i j} \frac{\partial^{2}}{\partial y_{i} \partial y_{j}}+\sum_{i} \mathscr{B}_{i} \frac{\partial}{\partial y_{i}}, \quad\left(y \in W_{0}\right)
$$

where the coefficients $\mathscr{A}_{i j}$ are $C^{p-1}$ and the $\mathscr{B}_{i}$ are $C^{p-2}$ in $y$.

Let $u$ be a generalized solution of $L u=\chi$, with $u \in H^{2, t}\left(W_{0}\right)$ for every $t>1$, while $\chi \in C^{q-2}\left(\overline{D_{n}}\right)$ and $\chi=0$ on $W_{0} \backslash D_{n}$. Then $u \in C^{p}\left(W_{0} \backslash D_{n}\right) \cup C^{q}\left(W_{0} \backslash D_{n}\right)$ and

$$
\|u\|_{C^{r \text { in }(p, q)\left(W_{0} \backslash D_{n}\right)}} \leqslant C\left(\|\chi\|_{C^{q-2}\left(D_{n}\right)}+\|u\|_{C^{0}\left(W_{0}\right)}\right),
$$

for some constant $C$.

In the proof of Lemma 5 we shall repeatedly use the following lemma (compare Friedman [14] Th. 19.5).

LEMMA $5^{*}$. Let $L u=\tilde{\chi}$ in an open domain $\Omega$, where $L$ is as above, $\tilde{\chi} \in C^{k-2}(\Omega)(k>2)$ and $u \in H^{2, t}(\Omega)$ for every $t>1 ; k \notin \mathbf{N}$. Then for every subdomain $\Omega^{\prime}$ with $\Omega^{\prime} \subset \Omega$ we have $u \in C_{\ell}\left(\Omega^{\prime}\right)$; $u \in H^{[\ell], t}\left(\Omega^{\prime}\right)$, where $\ell=\min (p, k)$, and

$$
\|u\|_{C^{\text {min }(p, h)(\Omega)}} \leqslant C\left(\|\tilde{\chi}\|_{C^{k}{ }_{2(\Omega)}}+\|u\|_{C^{0}(\Omega)}\right) \text {. }
$$

\section{Proof of Lemma 5.}

We introduce polar coordinates in $W_{0}$. The operator $L$ becomes

$$
L=a_{0}(r, \phi) \frac{\partial^{2}}{\partial r^{2}}+\sum_{i} a_{i}(r, \phi) \frac{\partial^{2}}{\partial r \partial \phi_{i}}+\sum_{i, j} a_{i j}(r, \phi) \frac{\partial^{2}}{\partial \phi_{i} \partial \phi_{j}}+b_{0}(r, \phi) \frac{\partial}{\partial r}+\sum_{i} b_{i}(r, \phi) \frac{\partial}{\partial \phi_{i}}
$$

here the $a$ 's are $C^{p-1}$ and the $b$ 's are $C^{p-2}$ on the domain $\dot{W}_{0}=W_{0} \backslash\{0\}$. Moreover, $a_{0} \in C^{p-1}\left(W_{0}\right)$, 
$a_{0}>0$ in $W_{0}$, the $a_{i}(i>0)$ and $b_{0}$ may have a singularity of order $1 / r$ at 0 and the $a_{i j}$ and $b_{i}(i>0)$ may have a singularity of order $1 / r^{2}$ at 0 .

If $p$ or $q<3$, Lemma $5^{*}$ immediately proves Lemma 5 .

If $p$ and $q>3$, Lemma $5^{*}$ gives $u \in C^{3-}\left(W_{0}\right)$ and for every $\Omega^{1}$ with $\Omega^{1} \subset \dot{W}_{0}\left(=W_{0} \backslash\{0\}\right)$,

$$
\|u\|_{C^{2+\alpha_{\left(\Omega^{1}\right)}}} \leqslant C\left(\|\chi\|_{C^{q-2}\left(D_{n}\right)}+\|u\|_{C^{0}\left(W_{0}\right)}\right)
$$

for every $\alpha, 0<\alpha<1$; and for arbitrary $t>1$ :

$$
u \in H^{3, t}\left(W_{0}\right)
$$

Because now $u \in C^{3}\left(W_{0} \backslash \overline{D_{n}}\right)$ and $u \in C^{3}\left(D_{n}\right)$, we can differentiate both sides of $L u=\chi$ with respect to $\phi_{k}(k$ arbitrary, $1 \leqslant k \leqslant n-1)$ to obtain

$$
L \frac{\partial u}{\partial \phi_{k}}=\frac{\partial \chi}{\partial \phi_{k}}+N_{k}^{(1)} u
$$

for each $(r, \phi) \in W_{0} \backslash \partial D_{n}$, where

$$
N_{k}^{(1)}=-\left(\frac{\partial a_{0}}{\partial \phi_{k}} \frac{\partial^{2}}{\partial r^{2}}+\sum_{i} \frac{\partial a_{i}}{\partial \phi_{k}} \frac{\partial^{2}}{\partial r \partial \phi_{i}}+\ldots\right),
$$

so

$$
N_{k}^{(1)} u \in C^{p-3}\left(\dot{W}_{0}\right) \cup C^{1-}\left(\Omega^{1}\right)
$$

We have for $\alpha<1, \alpha \leqslant p-3$ :

$$
\left\|N_{k}^{(1)} u\right\|_{C^{\alpha}\left(\Omega^{1}\right)} \leqslant C\|u\|_{C^{2+\alpha}\left(\Omega^{1}\right)} \leqslant C\left(\|\chi\|_{C^{q-2}\left(D_{n}\right)}+\|u\|_{C^{0}\left(W_{0}\right)}\right)
$$

and

$$
\frac{\partial \chi}{\partial \phi_{k}} \in C^{q-3}\left(\Omega^{1}\right)
$$

and

$$
N_{k}^{(1)} u \in H^{1, t}\left(\Omega^{1}\right)
$$

So with (3.51) and Lemma $5^{*}$ we find for each $\overline{\Omega^{2}}$ with $\Omega^{2} \subset \Omega^{1}$ :

$$
\frac{\partial u}{\partial \phi_{k}} \in C^{p-1}\left(\Omega^{2}\right) \cup C^{q-1}\left(\Omega^{2}\right) \cup C^{3-}\left(\Omega^{2}\right)
$$

and

$$
\left\|\frac{\partial u}{\partial \phi_{k}}\right\|_{C^{2+\alpha\left(\Omega^{2}\right)}} \leqslant C\left(\|\chi\|_{C^{q-2}\left(D_{n}\right)}+\|u\|_{C_{\left(W_{0}\right)}}\right)
$$

for $0<\alpha \leqslant \min (p-3, q-3), \alpha<1$.

If $p$ and $q$ are both $>4$, then $\left(\partial u / \partial \phi_{k}\right) \in H^{3, t}\left(\Omega^{2}\right)$ and $\left(\partial u / \partial \phi_{k}\right) \in C^{3-}\left(\Omega^{2}\right)$ and (3.58) is valid for all $\alpha, 0<\alpha<1$. 
We can now proceed by induction; we find that for every multi-index $m$ with $|m|<\min (p-2$, $q-2$ ), we have

$$
L \frac{\partial^{|m|} u}{\partial \phi_{1}^{m_{1}} \ldots \partial \phi_{n-1}^{m_{n-1}}}=\frac{\partial^{|m|}}{\partial \phi_{1}^{m_{1}} \ldots \partial \phi_{n-1}^{m_{n-1}}}+N_{m_{1} \ldots m_{n-1}}^{(|m|)} u,
$$

(which, in fact, defines $N_{m_{1} \ldots m_{n-1}}^{(|m|)}$ ); we can prove

$$
\begin{gathered}
\left|N_{m_{1} \ldots m_{n-1}}^{(|m|)} u\right|_{C^{\alpha}\left(\Omega^{(m))}\right.} \leqslant C\left(\|\chi\|_{C^{a-2}\left(D_{n}\right)}+\|u\|_{C^{0}\left(W_{0}\right)}\right), \\
N_{m_{1} \ldots m_{n-1}}^{(|m|)} u \in H^{1, t}\left(\Omega^{(m)}\right) \\
\frac{\partial^{|m|}}{\partial \phi_{1}^{m_{1}} \ldots \partial \phi_{n-1}^{m_{n-1}}} \in C^{q-|m|-2}\left(\Omega^{(m)}\right)
\end{gathered}
$$

and

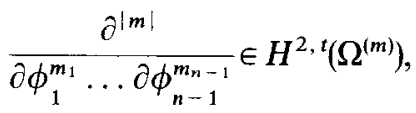

so with Lemma $5^{*}$ we conclude that all derivatives, with respect to the variables $\phi$ of order $<\min (p-2, q-2)$, are at least in $C^{2}\left(\dot{W}_{0}\right)$, so the derivatives of type

$$
\frac{\partial^{\prime}}{\partial r^{\prime}} \frac{\partial^{|m|}}{\partial \phi_{1}^{m_{1}} \ldots \partial \phi_{n-1}^{m_{n-1}}}
$$

with $\ell, m_{1} \ldots m_{n-1} \in \mathbf{N}, \ell+|m|<p, \ell+|m|<q$ and $\ell \leqslant 2$ are continuous across $\partial D_{n}$, and

$$
\left\|\frac{\partial^{\ell+|m|}}{\partial r^{l} \partial \phi_{1}^{m_{1}} \ldots \partial \phi_{n-1}^{m_{n-1}}}\right\|_{C^{0^{\prime}\left(\Omega_{0}\right)}} \leqslant C\left(\|u\|_{C^{0}\left(W_{0}\right)}+\|\chi\|_{C^{q-2}\left(D_{n}\right)}\right),
$$

for every $\Omega_{0}$ with $\overrightarrow{\Omega_{0}} \subset \dot{W}_{0}$.

The highest derivatives $(\ell+|m|=[p]$ or $[q])$ are even Höldercontinuous with exponent $p-[p]$ or $q-[q]$.

We now turn our attention to the derivatives with $\ell>2$. On $W_{0} \backslash D_{n}$ we have $L u=0$, so

$$
\frac{\partial^{2} u}{\partial r^{2}}=D_{1}^{(2)} \frac{\partial u}{\partial r}+D_{0}^{(2)} u
$$

where

$$
D_{1}^{(2)}=-\frac{1}{a_{0}}\left(\sum_{i} a_{i} \frac{\partial}{\partial \phi_{i}}+b_{0}\right)
$$

and

$$
D_{0}^{(2)}=-\frac{1}{a_{0}}\left(\sum_{i} b_{i} \frac{\partial}{\partial \phi_{i}}+\sum_{i, j} a_{i j} \frac{\partial}{\partial \phi_{i}} \frac{\partial}{\partial \phi_{j}}\right) .
$$

For $3<\min (p, q)<4$ the right member of $(3.64)$ is in $C^{p-2}\left(W_{0} \backslash D_{n}\right) \cup C^{q-2}\left(W_{0} \backslash D_{n}\right)$ so $\left(\partial^{3} u / \partial r^{3}\right)$ is in $C^{p-3}\left(W_{0} \backslash D_{n}\right) \cup C^{q-3}\left(W_{0} \backslash D_{n}\right)$, and

$$
\begin{aligned}
\left.\left\|\frac{r^{3} u}{\partial r^{3}}\right\|\right|_{\left(\min (p-3, q-3)\left(\boldsymbol{W}_{0}\right) \boldsymbol{D}_{n}\right)} & \leqslant C\left(\left\|D_{1}^{(2)} \frac{\partial u}{\partial r}\right\|^{(2)}+\left\|D_{0}^{(2)} u\right\|\right) \\
& \leqslant C\left(\|\chi\|_{C^{q-2}\left(D_{n}\right)}+\|u\|_{C^{0}\left(\boldsymbol{W}_{0}\right)}\right)
\end{aligned}
$$


we can write

$$
\frac{\partial^{3} u}{\partial r^{3}}=\left(\frac{\partial}{\partial r} D_{1}^{(2)}\right) \frac{\partial u}{\partial r}+D_{1}^{(2)} \frac{\partial^{2}}{\partial r^{2}} u+\left(\frac{\partial}{\partial r} D_{0}^{(2)}\right) u+D_{0}^{(2)} \frac{\partial u}{\partial r},
$$

where $(\partial / \partial r) D_{i}^{(2)}$ is the operator derived from $D_{i}^{(2)}$ by differentiating its coefficients with respect to $r$. With (3.64) we can reduce the $\left(\partial^{2} u / \partial r^{2}\right)$ term to derivatives of order 1 and 0 , so

$$
\frac{\partial^{3} u}{\partial r^{3}}=D_{1}^{(3)} \frac{\partial}{\partial r} u+D_{0}^{(3)} u
$$

where now $D_{i}^{(3)}$ is a differential operator of order $3-i$ with coefficients in $C^{p-3}\left(W_{0} \backslash D_{n}\right)$.

For $4<\min (p, q)<5$ we have the right member of $(3.69)$ in $C^{p-3}\left(W_{0} \backslash D_{n}\right) \cup C^{q-3}\left(W_{0} \backslash D_{n}\right)$ so $\left(\partial^{4} u / \partial r^{4}\right)$ and $\left(\partial^{4} u / \partial r^{3} \partial \phi_{k}\right)$ are in $C^{p-4}\left(W_{0} \backslash D_{n}\right) \cup C^{q-4}\left(W_{0} \backslash D_{n}\right)$, with corresponding estimates of the form (3.67).

More generally, by induction we obtain

$$
\frac{\partial^{k} u}{\partial r^{k}}=D_{1}^{(k)} \frac{\partial u}{\partial r}+D_{0}^{(k)} u
$$

where

$$
D_{j}^{(k)}=\sum_{1 \leqslant|m| \leqslant k} \pi_{m k j} \frac{\partial^{|m|}}{\partial \phi_{1}^{m_{1}} \cdots \partial \phi_{n-1}^{m_{n-1}}},
$$

the $\pi_{m k j}$ are real coefficients with a singularity in 0 of order $1 / r^{|m|}$ or less, and

$$
\pi_{m k j} \in C^{p-k+j-1+|m|}\left(\dot{W}_{0}\right)
$$

The assertion of Lemma 5 is now easily obtained.

Remark. If $L$ satisfies the conditions of Lemma 5 and if $u$ is the generalized solution in $H^{2, t}$ of $L u=\partial \chi / \partial r$, where $\chi \in C^{q-1}\left(\overline{D_{n}}\right)$ and $\chi=0$ on $W_{0} \backslash \overline{D_{n}}$, we can prove $u \in C^{p-1}\left(W_{0} \backslash D_{n}\right) \cup C^{q-1}\left(W_{0} \backslash\right.$ $\left.D_{n}\right)$.

We can use Lemma 5 to find a regularity result for $f(\varepsilon, \rho, y)$. Applying the lemma to the problem

$$
\begin{aligned}
& L u_{\mathrm{pre}}^{*}+\lambda U=0 \quad\left(\text { in } D_{n}\right), \\
& L u_{\mathrm{pre}}^{*}=0 \quad\left(\text { in } W_{0} \backslash D_{n}\right),
\end{aligned}
$$

(as obtained from (3.42), where $L$ is defined by (3.30) and $u_{\mathrm{pre}}^{*}(y)=u_{\mathrm{pre}}\left(T_{\sigma(\tilde{\gamma}(\varepsilon))}(y)\right)$, we find $u_{\mathrm{pre}} \in$ $C^{p}(\Omega \backslash \tilde{\omega}(\varepsilon))$; hence, $f \in C^{p}\left(\partial D_{n} ; \mathbf{R}\right)$.

We have proved:

Corollary. $f$ is a mapping in $C^{p}\left(\partial D_{n}\right)$.

(ii) the $\varepsilon$-dependence of $f$.

Using the Green's function representation of $\mathscr{L}^{-1}$ we can write

$$
\begin{aligned}
F(\varepsilon, \rho, y) & =\int_{\omega(\varepsilon)} G\left(T_{\tau(\hat{\gamma}(\xi))}(y), \xi\right) u_{i n}(\varepsilon, \rho, \xi) \mathrm{d} \xi \\
& =\int_{D_{n}} G\left(T_{\sigma(\hat{\gamma}(\varepsilon))}(y), T_{\sigma(\hat{\gamma}(i))}(\eta)\right) U(\varepsilon, \rho, \eta) J(\varepsilon, \rho, \eta) \mathrm{d} \eta
\end{aligned}
$$


where

$$
J=\operatorname{det}\left(\frac{\partial\left(T_{\sigma(\hat{\gamma(\varepsilon))}}(\eta)\right)_{i}}{\partial \eta_{j}}\right)=\sum_{m \geqslant 0} \varepsilon^{m} J_{m}(\rho, \eta)
$$

with $J_{m} \in C^{p-1}, J_{m} m$-homogeneous in $\rho$ and $\left\|J_{m}\right\|_{p-1} \leqslant\|\rho\|_{p}^{m} ; U$ still is the function defined in (3.29).

LEMMA 6. Let $k<\min \left(p_{0}, p+1\right)$ and let $W_{0}^{1} \subset W_{0}$ be a bounded neighbourhood of $D_{n}$. There is a bounded neighbourhood $C$ of 0 in $C^{p}\left(\partial D_{n} ; \mathbf{R}\right)$ and an $\varepsilon_{0}>0$ such that the first $k-1$ derivatives of $\mathscr{F}$ with respect to $\varepsilon$ are uniformly bounded on $\left[0, \varepsilon_{0}\right] \times C \times W_{0}^{1} \backslash \bar{D}_{n}$; moreover, these derivatives can be continuously extended to $\left[0, \varepsilon_{0}\right] \times C \times W_{0}^{1} \backslash D_{n}$.

Proof. Take $C$ and $\varepsilon_{0}$ such that $1+\varepsilon \rho$ maps $\partial D_{n}$ into $T_{0} W_{0}$ and $\|\varepsilon \rho\|_{p}<1$ for all $\varepsilon \in\left[0, \varepsilon_{0}\right]$ and $\rho \in C$.

The $m^{\text {th }}$ derivative of $\mathscr{F}$ with respect to $\varepsilon$ is

$$
\sum_{\nu=0}^{m}\left(\begin{array}{c}
m \\
v
\end{array}\right) \int_{D_{n}} \frac{\partial^{v}}{\partial \varepsilon^{v}}\left\{G\left(T_{\sigma(\tilde{\gamma}(\varepsilon))}(y) ; T_{\sigma(\bar{\gamma}(\varepsilon))}(\eta)\right)\right\} \frac{\partial^{m-v}}{\partial \varepsilon^{m-\nu}}\{U J\} \mathrm{d} \eta
$$

We first study the derivative of Green's function:

$$
\frac{\partial^{v}}{\partial \varepsilon^{v}}\left\{G\left(T_{\sigma(\tilde{\gamma}(\varepsilon))}(y) ; T_{\sigma(\hat{\gamma} \varepsilon))}(\eta)\right)\right\}=\frac{\partial^{v}}{\partial \varepsilon^{v}}\{H((s(1+\varepsilon \rho(\psi)), \psi) ;(r(1+\varepsilon \rho(\phi)), \phi))\},
$$

where

and

$$
H(u, v)=G\left(T_{0}(u), T_{0}(v)\right), \quad(u, v) \in W_{0} \times W_{0}
$$

$$
y=(s, \psi), \eta=(r, \phi) \quad\left(\text { polar coordinates on } W_{0}\right)
$$

So, (3.76) becomes

$$
\sum_{i=0}^{v}\left(\begin{array}{l}
v \\
i
\end{array}\right) \frac{\partial^{i}}{\partial s^{i}} \frac{\partial^{v-i}}{\partial r^{\nu-i}} H((s(1+\varepsilon \rho), \psi) ;(r(1+\varepsilon \rho), \phi)) \cdot\left(\frac{s \rho(\psi)}{1+\varepsilon \rho(\psi)}\right)^{i}\left(\frac{r \rho(\phi)}{1+\varepsilon \rho(\phi)}\right)^{v-i}
$$

Now $\mathscr{L} G(x ; \xi)=0$ both in the variables $x$ and $\xi$ (not when $x=\xi$, which does not occur because $y=T_{\sigma(\hat{\gamma}(\varepsilon))}^{-1}(x) \notin D_{n}$ ), so using the samc transformation on polar coordinates as in the proof of Lemma 5 we find:

$$
\frac{\partial i}{\partial s^{i}} \frac{\partial^{v-i}}{\partial r^{\nu-i}} H=\frac{\partial^{i}}{\partial s^{i}}\left(D_{1}^{(v-i)} \frac{\partial H}{\partial r}+D_{0}^{(\nu-i)} H\right)
$$

where $D_{j}^{v-i}$ is a differential operator in the variable $\phi$, defined by (3.71).

The $m^{\text {th }}$ derivative of $\mathscr{F}(3.75)$, turns out to consist of terms

with

$$
\frac{\partial^{i}}{\partial s^{i}} \int_{D_{n}} D_{1}^{(v-i)} \frac{\partial H}{\partial r} I_{i}(\varepsilon, \rho,(r, \phi)) \mathrm{d} r \mathrm{~d} \phi+\frac{\partial}{\partial s^{i}} \int_{D_{n}} D_{0}^{(v-i)} H I_{i}(\varepsilon, \rho,(r, \phi)) \mathrm{d} r \mathrm{~d} \phi,
$$

$$
I_{i}=\frac{\partial^{m-v}}{\partial \varepsilon^{m-v}} U((\varepsilon, \rho,(r, \phi)) J(\varepsilon, \rho,(r, \phi)))\left(\frac{s \rho(\psi)}{1+\varepsilon \rho(\psi)}\right)^{i}\left(\frac{r \rho(\phi)}{1+\varepsilon \rho(\phi)}\right)^{v-i}
$$


so $I_{i}$ is a $C^{p-1}$ function of $(r, \phi),\left\|I_{i}\right\|_{p-1}$ is bounded on $\left[0, \varepsilon_{0}\right] \times C \times W_{0} \backslash D_{n}, I_{i}=0$ for $\eta \in \partial D_{n}, I_{i}$ has a zcro of order $v-i+f$ in $r=0$. These zero's appear to annihilate the singularities in the coefficients of $D_{i}^{(v-i)}$ and $D_{0}^{(v-i)}$, so (3.81) equals

$$
-\frac{\partial^{i}}{\partial s^{i}} \int_{D_{n}} H \frac{\partial}{\partial r} D_{1}^{(v-i)^{*}} I_{i}(\varepsilon, \rho,(r, \phi)) \mathrm{d} r \mathrm{~d} \phi+\frac{\partial i}{\partial s^{i}} \int_{D_{n}} H D_{0}^{(v-i)^{*}} I_{i}(\varepsilon, \rho,(r, \phi)) \mathrm{d} r \mathrm{~d} \phi,
$$

where $D_{j}^{(v-i)^{*}}$ is the adjoint of $D_{j}^{(v-i)}$; it is also of the form $(3.71)$, but now the coefficients are only in $C^{p_{0}-(v-i)+j-1}$.

Let $v$ be the solution of

$$
\begin{array}{rlrl}
\mathscr{L} v & =D_{0}^{(v-i)^{*}} I_{i}\left(\varepsilon, \rho, T_{0}^{-1}(\xi)\right) . J_{0}^{-1} & \left(\xi \in \omega_{0}\right), \\
& =0 & & \left(\text { in } \Omega \backslash \omega_{0}\right), \\
v & =0 & & \text { (on } \partial \Omega) ;
\end{array}
$$

the conditions of Lemma 5 are satisfied (with $q-2=p-v+i-1$ ), so $v \in C^{p_{0}}\left(W_{0}^{1} \backslash D_{n}\right) \cup$ $C^{p-v+1+i}\left(W_{0}^{1} \backslash D_{n}\right)$. The second term of (3.83) now equals $-\partial^{i} v / \partial s^{i}\left(T_{0}^{-1}(s, \psi)\right)$, so this second term is certainly in $C^{p_{0}-i} \cup C^{p-v+1}$. The first term equals $-\partial^{i} / \partial s^{i} v\left(T_{0}^{-1}(s, \psi)\right)$ where

$$
\begin{array}{rlrl}
\mathscr{L} v & =-(\partial / \partial r)\left(D_{1}^{(v-i)^{*}} I_{i}\left(\varepsilon, \rho, T_{0}^{-1}(\xi)\right)\right) . J_{0}^{-1}\left(\xi \in \omega_{0}\right), \\
& =0 & & \left(\text { in } \Omega \backslash \omega_{0}\right), \\
v & =0 & & \text { (on } \partial \Omega) ;
\end{array}
$$

this $v$ will be in $C^{p-v+i}\left(W_{0}^{1} \backslash D_{n}\right) \cup C^{p_{0}-1}\left(W_{0}^{1} \backslash D_{n}\right)$, from the remark following Lemma 5. So the first term is in $C^{p_{0}-i-1} \cup C^{p-v}$, and with $v \leqslant m \leqslant k<\min \left(p, p_{0}-1\right)$ we prove that (3.81) and, hence, (3.75) is continuous on $\left[0, \varepsilon_{0}\right] \times C \times W_{0} \backslash D_{n}$; the boundedness of (3.81) and (3.75) follows from the boundedness of the $I_{i}(3.82)$ and from Lemma 5. (The derivatives of $F$ with respect to $\varepsilon$ of order up to $k-1$ are even Höldercontinuous.)

Corollary to lemma 6.

$$
f(\varepsilon, \rho, y)=\sum_{m=0}^{k-1} \varepsilon^{m} f_{m}(\rho, y)+R_{f, k}
$$

with $R_{f, k}=o\left(\varepsilon^{k-1}\right)$ uniformly on $C \times \partial D_{n}$.

Proof of the Corollary. Let $f(\varepsilon, \rho, y)$ be the extension of $\mathscr{F}(\varepsilon, \rho, y)$ to $\partial D_{n}$; Let $\mathscr{F}_{m}$ be the $m^{\text {th }}$ derivative of $\mathscr{F}$ with respect to $\varepsilon$ taken $\varepsilon=0$ and let $f_{m}(\rho, y)=F_{m}(\rho, y)$ be the extension of $\mathscr{F}_{m}$ to $\partial D_{n}$. Let $y_{0} \in \partial D_{n}$ and $\varepsilon \in\left[0, \varepsilon_{0}\right]$; let $M_{m} m$ ! be the bound on the $m^{\text {th }}$ derivative of $\mathscr{F}$ with respect to $\varepsilon$ and choose $y$ such that

$$
\left|\mathscr{F}(\varepsilon, \rho, y)-\mathscr{F}\left(\varepsilon, \rho, y_{0}\right)\right|<\tilde{M} \varepsilon^{k},
$$

then

$$
\begin{aligned}
f\left(\varepsilon, \rho, y_{0}\right)= & \sum_{m=0}^{k-1} \varepsilon^{m} f_{m}\left(\rho, y_{0}\right)+\mathscr{F}\left(\varepsilon, \rho, y_{0}\right)-\mathscr{F}(\varepsilon, \rho, y)+\mathscr{F}(\varepsilon, \rho, y)-\sum_{m=0}^{k-1} \varepsilon^{m} \mathscr{F}_{m}(\rho, y) \\
& +\sum_{m=0}^{k-1} \varepsilon^{m}\left(\mathscr{F}_{m}(\rho, y)-\mathscr{F}_{m}\left(\rho, y_{0}\right)\right)
\end{aligned}
$$


so

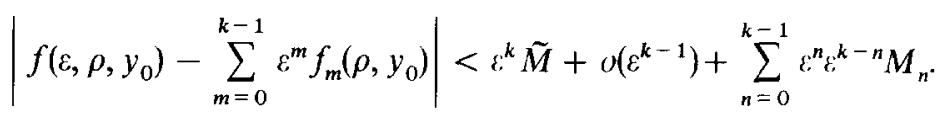

We calculate $f_{1}$ :

$$
\begin{aligned}
f_{1}(\rho,(s, \psi)) & =\int_{D_{n}} \frac{\partial}{\partial s} H((s, \psi) ;(r, \phi)) s \rho(\psi) U_{0}(r, \phi) J_{0}(r, \phi) \mathrm{d}(r, \phi) \\
& +\int_{D_{n}} \frac{\partial}{\partial r} H((s, \psi) ;(r, \phi)) r \rho(\phi) U_{0}(r, \phi) J_{0}(r, \phi) \mathrm{d}(r, \phi) \\
& +\int_{D_{n}} H((s, \psi) ;(r, \phi)) U_{1}(\rho,(r, \phi)) J_{0}(r, \phi) \mathrm{d}(r, \phi) \\
& +\int_{D_{n}} H((s, \psi) ;(r, \phi)) U_{0}(r, \phi) J_{1}(\rho,(r, \phi)) \mathrm{d}(r, \phi) .
\end{aligned}
$$

LEMMA 7. For $p<p_{0}-1$, the linear operator $f_{1}$ maps $C^{p}\left(\partial D_{n}\right)$ to itself. Furthermore,

$$
f_{1}(\rho, \psi)=c(\psi) \rho(\psi)+(\mathscr{A} \rho)(\psi)
$$

where $\mathscr{A}$ is a compact operator from $C^{p}$ to itself and $c(\psi) \in C^{p_{0}-1}\left(\partial D_{n} ; \mathbf{R}\right) ; c$ is uniformly positive on $\partial D_{n}$.

Proof. (3.92) leads to

$$
c(\psi)=\left.\int_{D_{n}} \frac{\partial}{\partial s} H((s, \psi) ;(r, \rho)) U_{0}(r, \phi) J_{0}(r, \phi) \mathrm{d}(r, \phi)\right|_{s=1},
$$

and

$$
\mathscr{A} \rho(\psi)=\lim _{s \downarrow 1}\left\{\left(\mathscr{A}_{1} \rho\right)(s, \psi)+\left(\mathscr{A}_{2} \rho\right)(s, \psi)+\left(\mathscr{A}_{3} \rho\right)(s, \psi)\right\}
$$

with

$$
\begin{aligned}
\left(\mathscr{A}_{1} \rho\right)(s, \psi) & =\int_{D_{n}}\left\{\frac{\partial}{\partial r} H((s, \psi) ;(r, \phi)) r U_{0}(r, \phi) J_{0}(r, \phi)\right\} \rho(\phi) \mathrm{d}(r, \phi), \\
\left(\mathscr{A}_{2} \rho\right)(s, \psi) & =\int_{D_{n}}\left\{H((s, \psi) ;(r, \phi)) J_{0}(r, \phi)\right\} U_{1}(\rho, r, \phi) \mathrm{d}(r, \phi), \\
\left(\mathscr{A}_{3} \rho\right)(s, \psi) & =\int_{D_{n}}\left\{H((s, \psi) ;(r, \phi)) U_{0}(r, \phi)\right\} J_{1}(\rho, r, \phi) \mathrm{d}(r, \phi) .
\end{aligned}
$$

Clearly, $c(\psi)$ is the outward normal derivative of $v(s, \psi)$ taken in a point $(1, \psi)$ of $\partial D_{n}$, where $w(x)=v\left(T_{0}^{-1}(x)\right)$ satisfies

$$
\begin{aligned}
\mathscr{L} w & =U_{0}\left(T_{0}^{-1}(x)\right) & & \left(x \in \omega_{0}\right), \\
\mathscr{L} w & =0 & & \left(x \in \Omega \backslash \omega_{0}\right), \\
w & =0 & & \text { (on } \partial \Omega) .
\end{aligned}
$$


Because $\omega_{0} \in G_{p_{0}}$, from Lemma 5 we find $w \in C^{p_{0}}\left(\Omega \backslash \omega_{0}\right)$, hence, $c$ is in $C^{p_{0}-1}$. Furthermore, the $T_{0}$-image of the normal derivative in $(1, \psi)$ cannot be tangential to $\partial \omega_{0}$ in $T_{0}(1, \psi)$ because $T_{0}^{\prime}$ is non-singular (see Definition 1), so using the strong maximum principle we find that $c(\psi)>0$ in each point of $\partial D_{n}$. Compactness of $\partial D_{n}$ leads to $c \geqslant \varepsilon>0$ on $\partial D_{n}$.

We now turn to $\mathscr{A}$.

$\left(\mathscr{A}_{2} \rho\right)(s, \psi)$ equals $w\left(T_{0}^{-1}(x)\right)$ if $w(x)$ satisfies

$$
\begin{aligned}
\mathscr{L} w & =U_{1}\left(\rho, T_{0}^{-1}(x)\right) & & \text { in } \omega_{0}, \\
\mathscr{L} w & =0 & & \text { in } \Omega \backslash \omega_{0}, \\
w & =0 & & \text { on } \partial \Omega .
\end{aligned}
$$

Because $\rho \in C^{p}, U_{1} \in C^{p}$ so Lemma 5 gives $w \in C^{p+2}\left(\Omega \backslash \omega_{0}\right) \cup C^{p_{0}}\left(\Omega \backslash \omega_{0}\right)$ and because $p+1<p_{0}$, then certainly $w \in C^{p+1}\left(\Omega \backslash \omega_{0}\right)$. Furthermore, $\|w\|_{p+1} \leqslant C\|\rho\|_{p}$, so $\mathscr{A}_{2}$ is a bounded mapping from $C^{p}$ to $C^{p+1}$. Now from Ascoli's theorem, $C^{p+1}$ is compactly imbedded in $C^{p}$, so $\mathscr{A}_{2}$ is a compact mapping in $C^{p}$.

The same kind of arguments apply to $\mathscr{A}_{3}$ and, after partial integration with respect to $r$, and recalling the remark following Lemma 5, we find that $A_{1}$ maps $C^{p}$ to $C^{p+1} \cup C^{p_{0}-1}$. Now using the Schauder estimate in Ladyzenskaya-Uralceva [16] p. 109 we obtain that also $A_{1}$ maps $C^{p}$ compactly to itself.

\subsection{Derivation of the bifurcation value and the bifurcating branch from the interface condition}

Theorem. Let $\min (n / 2,2) \leqslant p \leqslant p_{0}, 2 \leqslant k \leqslant \min \left(\left[p_{0}\right],[p]+1\right), p, p_{0} \notin \mathbf{N}$. Then a necessary condition for $\lambda_{0}$ to be a bifurcation value of type $(p, k)$ is that $0 \in \sigma\left(f_{1}\right)$.

Proof. Let $\lambda_{0}$ be such that $0 \notin \sigma\left(f_{1}\right)$.

From the Corollary to Lemma 6 , the branch $\tilde{\gamma}(\varepsilon)=1+\varepsilon \rho$ must satisfy

$$
f_{0}+\varepsilon f_{1}(\rho)+R_{f, 2}(\rho, \varepsilon)=\alpha(\varepsilon) .1 \text { on } \partial D_{n},
$$

where $\alpha(\varepsilon)$ is some real scalar function on the set of small $\varepsilon$.

By applying the implicit function theorem, we find

$$
\varepsilon \rho=\left(\alpha-f_{0}\right) K(\mathbf{1})+O\left(\varepsilon,\left|\alpha-f_{0}\right|\right),
$$

where $K$ is the inverse of $f_{1}$. If we now identify the free parameter $\varepsilon$ with the function $\alpha-f_{0}$, then $\rho(\varepsilon)-K(1)+O(\varepsilon)$ and from (3.28) $\lambda=\lambda(\varepsilon, \rho)=\lambda(\varepsilon, \rho(\varepsilon))$. Now $\rho$ and $\lambda$ are uniquely determined functions of the parameter $\varepsilon$. These unique solutions, however, represent the variation of the basic branch, so $\lambda_{0}$ cannot be a bifurcation value.

We shall now investigate what happens if $0 \in \sigma\left(f_{1}\right)$, using Fredholm-Riesz-Schauder theory (see e.g. Sattinger [11], p. 49 v.v.).

Because $\mathscr{A}$ is compact, $0 \in \sigma\left(f_{1}\right)$ implies that $\operatorname{Ker}\left(I+c^{-1} \mathscr{A}\right)$ and, hence, $\operatorname{Ker}(c I+\mathscr{A})$ is at least one-dimensional ( $I$ is the identity: $C^{p}\left(\partial D_{n}\right) \rightarrow C^{p}\left(\partial D_{n}\right)$ ). So 0 is an eigenvalue of $f_{1}$; assume it has multiplicity 1 , with eigenvector $\phi_{0}$. If we define $N=\operatorname{Ker}\left(I+c^{-1} \mathscr{A}\right)$ and $R=\operatorname{Range}$ $\left(I+c^{-1} \mathscr{A}\right)$ then $N$ and $R$ are invariant subspaces under $I+c^{-1} \mathscr{A}, C^{p}=N \oplus R$. Furthermore, a bounded 'pseudo inverse' $K: R \rightarrow R$ exists, with $K\left(I+c^{-1} \mathscr{A}\right) \psi=\left(I+c^{-1} \mathscr{A}\right) K \psi=\psi$ for all 
$\psi$ in $R$. Let $P: C^{p} \rightarrow N$ be the projection operator, then the equation

$$
f_{1} \chi=\Xi
$$

$\Xi \in C^{p}$, has a solution $\chi \in R$

$$
\chi=K\left(c^{-1} \Xi\right)
$$

if and only if

$$
P\left(c^{-1} \Xi\right)=0
$$

We now want to solve

$$
\varepsilon f_{1}(\rho)=\varepsilon b(\varepsilon)+\sum_{m=2}^{k-1} \varepsilon^{m} f_{m}(\rho)+R_{f},
$$

(where we have put $\alpha(c)=f_{0}+\varepsilon b(\varepsilon)$ ). We decomposc

$$
\rho=\kappa \phi_{0}+\psi
$$

Introducing this into (3.102) we obtain

$$
\psi=K\left(c^{-1} \sum_{m=2}^{k-1} \varepsilon^{m-1} f_{m}\left(\kappa \phi_{0}+\psi\right) f+c^{-1} R_{f, k} \varepsilon^{1}+c^{1} b\right)
$$

provided

$$
P\left(c^{-1} \sum_{m=2}^{k-1} \varepsilon^{m} f_{m}\left(\kappa \phi_{0}+\psi\right)+c^{-1} R_{f, k}+c^{-1} b \varepsilon\right)=0 .
$$

From (3.104) we can solve $\psi=\Psi(b, \varepsilon, \kappa)$ in the form

$$
\Psi(b, \varepsilon, \kappa)=\sum_{\substack{\ell, m \\ 1 \leqslant \ell+m \leqslant k-1}} \psi_{\ell m} \varepsilon^{\ell+m-1} \kappa^{m} b^{\ell}+O\left(\varepsilon^{k-2}\right),
$$

with

$$
\begin{array}{ll}
\psi_{10}=K\left(c^{-1} 1\right), & \psi_{02}=K\left(c^{-1} f_{2}\left(\phi_{0}, \phi_{0}\right)\right), \\
\psi_{01}=0, & \psi_{30}=K\left(c^{-1} f_{3}\left(\psi_{10}, \psi_{10}, \psi_{10}\right)\right), \\
\psi_{20}=K\left(c^{-1} f_{2}\left(\psi_{10}, \psi_{10}\right)\right), & \text { etc. } \\
\psi_{11}=K\left(\left(c^{-1} f_{2}\left(\phi_{0}, \psi_{10}\right)\right),\right. &
\end{array}
$$

We have used that $f_{m}$ (defined by 3.86 ) is a $m$-linear operator.

Introducing this into (3.105) we find

$$
P\left(\sum_{m=2}^{k-1} \varepsilon^{m} c^{-1} f_{m}\left(\kappa \phi_{0}+\Psi(b, \varepsilon, \kappa)\right)+c^{-1} R+b \varepsilon c^{-1}\right)=0
$$

which is, in fact, one equation for the three variables $\kappa, b$ and $\varepsilon$, and can also be written as follows:

$$
\sum_{\substack{\ell, m \\ 1 \leqslant \ell+m \leqslant k-1}} \mathscr{A}_{\ell m}(b \varepsilon)^{\ell}(\varepsilon \kappa)^{m}+\mathrm{O}\left(\varepsilon^{k-1}\right)=0,
$$


where

$$
\begin{array}{llrl}
\mathscr{A}_{10} & =P\left(c^{-1} 1\right), & \mathscr{A}_{11} & =P\left(c^{-1} f_{2}\left(\phi_{0}, \psi_{01}\right)\right), \\
\mathscr{A}_{01} & =0, & \mathscr{A}_{02} & =P\left(c^{-1} f_{2}\left(\psi_{01}, \psi_{01}\right)\right), \\
\mathscr{A}_{20} & =P\left(c^{-1} f_{2}\left(\phi_{0}, \phi_{0}\right)\right), & \mathscr{A}_{03} & =P\left(c^{-1} f_{3}\left(\phi_{0}, \phi_{0}, \phi_{0}\right)\right) .
\end{array}
$$

We are now going to solve (3.109) for $b$ in terms of $\varepsilon$ and $\kappa$, using the Newton Polygon method. Suppose that the following two hypotheses are fulfilled:

$H_{1}$. For the basic branch $\left\{\omega_{0}(\lambda), u_{0}(\lambda), \lambda\right\}$ passing through $\left(\omega_{0}\left(\lambda_{0}\right), u_{0}\left(\lambda_{0}\right), \lambda_{0}\right)$ (see (3.13)) the boundary $\gamma_{0}(\lambda)$ of $\omega_{0}(\lambda)$ is given by

$$
\gamma_{0}(\lambda)=1+\left(\lambda-\lambda_{0}\right) \rho_{0}+0\left(\lambda-\lambda_{0}\right)^{2} .
$$

Further assume that $\rho_{0}$ and $\phi_{0}$ are linearly independent.

$H_{2}$. There is an $r, 2 \leqslant r \leqslant k-1$ such that

$$
\begin{aligned}
& P\left(c^{-1} f_{2}\left(\phi_{0}, \phi_{0}\right)\right)=\ldots=P\left(c^{-1} f_{r-1}\left(\phi_{0}, \ldots, \phi_{0}\right)\right)=0, \\
& \text { and } P\left(c^{-1} f_{r}\left(\phi_{0}, \ldots, \phi_{0}\right)\right) \neq 0
\end{aligned}
$$

i.e. $\quad \mathscr{A}_{02}=\ldots=\mathscr{A}_{\mathrm{Or}-1}=0$ and $\mathscr{A}_{\mathrm{Or}} \neq 0$.

Furthermore, $\mathscr{A}_{11} \neq 0$ and $\mathscr{A}_{20} \neq 0$.

(These last assumptions are not essential but they simplify the analysis of the Newton Polygon. It will be clear from the sequel how to analyse the cases where these conditions are not fulfilled.)

Hypothesis $H_{1}$ implies that if we decompose $\rho_{0}=\kappa \phi_{0}+\psi$, then $\psi \neq 0$. Because $\rho_{0}$ has to satisfy (3.104) we find that the corresponding $b \neq 0$ and, hence, from (3.105) it follows that $P\left(c^{-1} 1\right)=0$. So we find from $H_{1}$ :

$$
\mathscr{A}_{10}=0 \text {. }
$$

Together with $\mathrm{H}_{2}$ this implies that the principal part of (3.109) can be written as

$$
(\varepsilon b)^{2} \mathscr{A}_{20}+(\varepsilon \kappa)(\varepsilon b) \mathscr{A}_{11}+(\varepsilon \kappa)^{r} \mathscr{A}_{0 r}+\ldots=0 \text {. }
$$

We have to distinguish two cases:

In case $r=2:(3.112)$ leads to the study of the quadratic equation

$$
x^{2} \mathscr{A}_{20}+x y \mathscr{A}_{11}+y^{2} \mathscr{A}_{02}=0
$$

which has 0,1 or 2 solutions. 0 solutions is impossible because we know that there is at least one branch of solutions through $\left\{\omega_{0}\left(\lambda_{0}\right), u_{0}\left(\lambda_{0}\right), \lambda_{0}\right\}$. We shall not consider the degenerate case of 1 root, so assume there are two roots:

$$
x=\mathscr{R}_{1} y \text { and } x=\mathscr{R}_{2} y .
$$

The solutions of (3.112) are then

with

$$
b_{i}=\sum_{m=1}^{k-1} \varepsilon^{m-1} \kappa^{m} b_{i}^{(m)}+\mathrm{o}\left(\varepsilon^{k-2}\right) \quad(i=1,2)
$$

and

$$
\mathrm{b}_{1}^{(1)}=\mathscr{R}_{1}, b_{2}^{(1)}=\mathscr{R}_{2} \text {, }
$$

$$
b_{1}^{(2)}=\mathscr{A}_{20}^{-1}\left(\mathscr{R}_{2}-\mathscr{R}_{1}\right)^{-1} \sum_{j=0}^{3} \mathscr{A}_{j 3-j} \mathscr{R}_{1}^{j} .
$$

In the sequel we let $b_{1}$ represent the basic solution and $b_{2}$ the bifurcating one. 
In case $r>2$ : the solutions of (3.112) are:

$$
\begin{aligned}
& b_{1}=\sum_{m=1}^{k-1} \varepsilon^{m-1} \kappa^{m} b_{1}^{(m)}+O\left(\varepsilon^{k-2}\right), \\
& b_{2}=\sum_{m=r-1}^{k-1} \varepsilon^{m-1} \kappa^{m} b_{2}^{(m)}+O\left(\varepsilon^{k-2}\right)
\end{aligned}
$$

with

$$
b_{1}^{(1)}=-\mathscr{A}_{11} \cdot \mathscr{A}_{20}^{-1} \quad \text { and } \quad b_{2}^{(r-1)}=-\mathscr{A}_{0 r} \mathscr{A}_{11}^{-1}
$$

Again $b_{1}$ represents the basic solution and $b_{2}$ the bifurcating one.

So in both cases we have accomplished the task of solving (3.109) for $b$ in terms of $\varepsilon$ and $\kappa$, and we can write with (3.103) and (3.106):

$$
\rho=\kappa \phi_{0}+\Psi(b(\varepsilon, \kappa), \varepsilon, \kappa)=\rho(\varepsilon, \kappa) .
$$

To obtain the bifurcating branch in terms of $\rho(\lambda)$ we now introduce $b_{2}$ (given by (3.113) if $r=2$ and else given by (3.114)) into (3.28). We obtain

$$
\lambda=\lambda\left(1+\varepsilon\left(\kappa \phi_{0}+\Psi(b(\varepsilon, \kappa), \kappa, \varepsilon)\right)\right)=\sum_{m=0}^{k-1} C_{m}(\varepsilon \kappa)^{m}+O\left(\varepsilon^{k-1}\right),
$$

with

$$
\begin{aligned}
& C_{0}=\lambda_{0}, C_{1}=\lambda_{1}\left(\phi_{0}+b^{(1)} \psi_{10}\right), \\
& C_{2}=\lambda_{2}\left(\phi_{0}+b^{(1)} \psi_{10}\right)+\lambda_{1}\left(\psi_{10} b^{(2)}+\psi_{02}\right) .
\end{aligned}
$$

Now it is important which is the first non-vanishing coefficient $C_{n}$ :

Case 1: if $C_{1} \neq 0$ choose $\varepsilon=\lambda-\lambda_{0}$; we find

$$
\kappa=\sum_{m=0}^{k-2} K_{m}\left(\lambda-\lambda_{0}\right)^{m}+O\left(\lambda-\lambda_{0}\right)^{k-2}
$$

with

$$
K_{0}-\left(C_{1}\right)^{-1}
$$

hence,

$$
\gamma=1+\left(\lambda-\lambda_{0}\right)\left(\left(C_{1}\right)^{-1} \phi_{0}+b^{(1)}\left(C_{1}\right)^{-1} \psi_{10}\right)+O\left(\lambda-\lambda_{0}\right)^{2} .
$$

Case 2a: if $C_{1}=0, C_{2}>0$ choose $\varepsilon=\left(\lambda-\lambda_{0}\right)^{1 / 2}$; we find

$$
\kappa=\sum_{m=0}^{k-3} K_{m}\left(\lambda-\lambda_{0}\right)^{m / 2}+O\left(\lambda-\lambda_{0}\right)^{(k-3) / 2},
$$

with

$$
K_{0}=\left(C_{2}\right)^{-1 / 2}
$$

hence,

$$
\text { for } \lambda>\lambda_{0} \text { : }
$$

$$
\gamma=1 \pm\left(\lambda-\lambda_{0}\right)^{1 / 2}\left(\left(C_{2}\right)^{-1 / 2} \phi_{0}+b^{(1)}\left(C_{2}\right)^{-1 / 2} \psi_{10}\right)+O\left(\lambda-\lambda_{0}\right) .
$$

Case 2b: if $C_{1}=0, C_{2}<0$ choose $\varepsilon=\left(\lambda_{0}-\lambda\right)^{1 / 2}$; we find $\left(\lambda<\lambda_{0}\right)$ :

$$
\gamma-1 \pm\left(\lambda_{0}-\lambda\right)^{1 / 2}\left(\left(-C_{2}\right)^{-1 / 2} \phi_{0}+b^{(1)}\left(-C_{2}\right)^{-1 / 2} \psi_{10}\right)+O\left(\lambda-\lambda_{0}\right) ; \text { etc. }
$$


THEOREM 2. Let $\min (n / 2,2) \leqslant p<p_{0}-1 ; k \leqslant \min \left(\left[p_{0}\right],[p]+1\right)$ and let $O$ be a simple eigenvalue of $f_{1}$, with $\phi_{0}$ the corresponding eigenvector; let $H_{1}$ and $H_{2}$ be fulfilled. If $k \geqslant 2$ and $C_{1} \neq 0\left(C_{1}\right.$ defined by (3.117)) then $\lambda_{0}$ is a bifurcation value of type $(p, k)$ and the (bilaterally) bifurcating branch is given by (3.118). If $k \geqslant 3$ and $C_{1}=0, C_{2} \neq 0$ then $\lambda_{0}$ is bifurcation value of type ( $p, k)$ and the (unilaterally) bifurcating branch is given by (3.119).

Remark. If all $\mathscr{A}_{0 r}$ vanish (because $H_{2}$ is not fulfilled), we may expect vertical bifurcation.

If $\mathscr{A}_{10} \neq 0$ (because $H_{1}$ is not fulfilled) the basic branch may have a turning point or a point of inflection. This can even occur at points $\lambda_{0}$ where $f_{1}$ is invertible, provided $\lambda_{1}\left(f_{1}^{-1}(1)\right)=0$. We shall not further analyse these points here because no real bifurcation takes place.

Acknowledgement-The author wishes to express his gratitude for the many stimulating discussions with Professors W. Eckhaus and W. M. Grcenlee.

\section{REFERENCES}

1. Mercier C., The magnetohydrodynamic approach to the problem of plasma confinement in closed magnetic configurations. Publ. Euratom C.E.A., Luxembourg (1974).

2. TEMam R., A nonlinear eigenvalue problem: the shape at equilibrium of a confined plasma Archs ration. Mech. Analysis 60, 51-73 (1976).

3. Temam R., Remarks on a frec boundary valuc problem arising in plasma physics, Communs part. diff. equns 2, 563-585 (1977).

4. SCHAEFFER D.G., Non uniqueness in the equilibrium shape of a confined plasma, Communs part. diff. equns 2, 587-600 (1977).

5. Puel J. P., Sur un problème de valeur propre non linéaire et de frontière libre, C.r. Acad. Sci., Paris 284A, 861-863 (1977).

6. KiNDERLEHRER D., Variational inequalities and free boundary problems, Bull. Am. math. Soc. 84, 7-26 (1978).

7. KINDERLEHRER D. \& SPRUCK J., The shape and smoothness of stable plasma configurations, Annali Scu. norm. sup., Pisa V, 131-149 (1978).

8. SijBrand J., Computation of the bifurcating solutions of a special class of problems with a free boundary, Report Math. Inst. Univ. Utrecht (1978).

9. BerestycKi H. \& BRÉzis H., Sur certains problèmes de frontière libre, C.r. Acad. Sci., Paris 283A, 1091 (1976).

10. StAKGOLD J., Branching of solutions of nonlinear equations, SIAM Rev. 13, 289-332 (1971).

11. Sattinger D. H., Topics in stability and bifurcation, Springer Lecture Notes in Math. 309 (1973).

12. Iooss G., Théorie non linéaire de la stabilité des écoulements laminaires dans le cas de l'échange des stabilités, Archs ration. Mech. Analysis 40, 166-208 (1971).

13. KATo T., Perturbation Theory of Linear Operators, Springer Verlag, Berlin (1966).

14. Friedman A., Partial Differential Equations, Holt, Rhinehart, Winston (1969).

15. Agmon S., Douglis A. \& Nirenberg L., Estimates near the boundary for solutions of elliptic partial differential equations satisfying general boundary conditions, I, Communs pure appl. Math. 12, 623-727 (1959).

16. Ladyženskaya O. A. \& Uralceva N. N., Equations aux Derivées Partielles de Type Elliptique. Dunod, Paris (1968) (Russian original 1964).

17. Mercier C. \& SoubBaramayer, Equilibre, perturbation et evolution d'un système plasma- vide du type Tokomak, preprint, Association Euraton-CEA, Fontenay aux Koses (1977).

18. Rellich F., Perturbation theory of eigenvalue problems. Inst. Math. Sci. New York Univ, Lecture Notes (1953). 